ANALYSIS \& PDE Volume $5 \quad$ No. $4 \quad 2012$ 3.

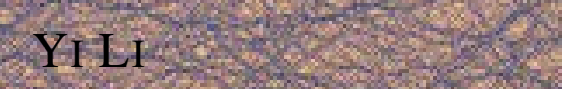

\title{
GENERALIZED RICCI FLOW
}

\section{I: HIGHER-DERIVATIVE ESTIMATES FOR COMPACT} MANIFOLDS 


\title{
GENERALIZED RICCI FLOW \\ I: HIGHER-DERIVATIVE ESTIMATES FOR COMPACT MANIFOLDS
}

\begin{abstract}
YI LI
We consider a generalized Ricci flow with a given (not necessarily closed) three-form and establish higher-derivative estimates for compact manifolds. As an application, we prove the compactness theorem for this generalized Ricci flow. Similar results still hold for a more generalized Ricci flow.

$\begin{array}{ll}\text { 1. Introduction } & 747\end{array}$

2. Short-time existence of GRF $\quad 752$

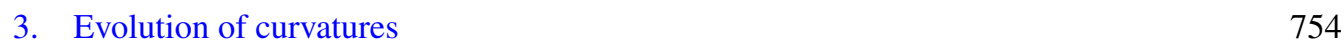

$\begin{array}{ll}\text { 4. Derivative estimates } & 756\end{array}$

5. Compactness theorem $\quad 764$

$\begin{array}{ll}\text { 6. Generalization } & 771\end{array}$

$\begin{array}{ll}\text { Acknowledgment } & 774\end{array}$

$\begin{array}{ll}\text { References } & 774\end{array}$
\end{abstract}

\section{Introduction}

Throughout this paper manifolds always mean smooth and closed (compact and without boundary) manifolds. Let $\operatorname{Met}(M)$ denote the space of smooth metrics on a manifold $M$, and $C^{\infty}(M)$ the set of all smooth functions on $M$. We denote by $C$ the universal constants depending only on the dimension of $M$, which may take different values at different places.

An important and natural problem in differential geometry is to find a canonical metric on a given manifold. A classical example is the uniformization theorem (e.g., [Chow and Knopf 2004]), which says that every smooth surface admits a unique conformal metric of constant curvature. To generalize to higher dimensional manifolds, Hamilton [1982] introduced a system of equations

$$
\frac{\partial g_{i j}}{\partial t}=-2 R_{i j}
$$

now called the Ricci flow, an analogue of the heat equation for metrics.

There are two ways to understand the Ricci flow: one way comes from the two-dimensional sigma model (see [Bakas 2007]), while another comes from Perelman's energy functional [Perelman 2002] defined by

$$
\mathscr{F}(g, f)=\int_{M}\left(R+|\nabla f|^{2}\right) e^{-f} d V_{g}, \quad(g, f) \in \mathfrak{M e t}(M) \times C^{\infty}(M),
$$

MSC2010: 53C44, 35K55.

Keywords: Ricci flow, Generalized Ricci flow, BBS derivative estimates, compactness theorems, energy functionals. 
where $R, \nabla$, and $d V_{g}$, is the scalar curvature, Levi-Civita connection, and volume form of $g$, respectively. He showed that the Ricci flow is the gradient flow of (1-2) and the functional $\mathscr{F}$ is monotonic along this gradient flow. Precisely, under the following system

$$
\frac{\partial g_{i j}}{\partial t}=-2 R_{i j}, \quad \frac{\partial f}{\partial t}=-R-\Delta f+|\nabla f|^{2},
$$

we have

$$
\frac{d}{d t} \mathscr{F}(g, f)=2 \int_{M}\left|R_{i j}+\nabla_{i} \nabla_{j} f\right|^{2} e^{-f} d V_{g} \geq 0 .
$$

Perelman's energy functional plays an essential role in determining the structures of singularities of the Ricci flow and then the proof of Poincaré conjecture and Thurston's generalization conjecture; for more details we refer readers to [Cao and Zhu 2006; Chow et al. 2006; 2007; 2008; 2010; Kleiner and Lott 2008; Morgan and Tian 2007; Perelman 2002].

Ricci flow coupled with a one-form or a two-form. If we consider the two-dimensional nonlinear sigma model [Bakas 2007; Oliynyk et al. 2006], then we obtain a generalized Ricci flow that is the Ricci flow coupled with the evolution equation for a two-form. This flow can be also obtained from the point of view of Perelman-type energy functional.

Denoting by $\mathscr{A}^{p}(M)$ the space of $p$-forms on $M$, we consider the energy functional

$$
\mathscr{F}^{(1)}: \operatorname{Met}(M) \times \mathscr{A}^{2}(M) \times C^{\infty}(M) \rightarrow \mathbb{R}
$$

defined by

$$
\mathscr{F}^{(1)}(g, B, f)=\int_{M}\left(R+|\nabla f|^{2}-\frac{1}{12}|H|^{2}\right) e^{-f} d V_{g},
$$

where $H=d B$. As showed in [Oliynyk et al. 2006], the gradient flow of $\mathscr{F}^{(1)}$ satisfies

$$
\begin{aligned}
\frac{\partial g_{i j}}{\partial t} & =-2 R_{i j}-2 \nabla_{i} \nabla_{j} f+\frac{1}{2} H_{i}{ }^{k \ell} H_{j k \ell}, \\
\frac{\partial B_{i j}}{\partial t} & =3 \nabla_{k} H^{k}{ }_{i j}-3 H^{k}{ }_{i j} \nabla_{k} f, \\
\frac{\partial f}{\partial t} & =-R-\Delta f+\frac{1}{4}|H|^{2},
\end{aligned}
$$

and under a family of diffeomorphisms the system (1-6)-(1-8) is equivalent to

$$
\begin{aligned}
\frac{\partial g_{i j}}{\partial t} & =-2 R_{i j}+\frac{1}{2} H_{i}{ }^{k \ell} H_{j k \ell}, \\
\frac{\partial B_{i j}}{\partial t} & =3 \nabla_{k} H^{k}{ }_{i j}, \\
\frac{\partial f}{\partial t} & =-R-\Delta f+|\nabla f|^{2}+\frac{1}{4}|H|^{2} .
\end{aligned}
$$

Using the adjoint operator $d^{*}$, Equation (1-10) can be written as

$$
\frac{\partial B_{i j}}{\partial t}=-\left(d^{*} H\right)_{i j},
$$


and therefore (because of $H=d B$ )

$$
\frac{\partial H}{\partial t}=-d d^{*} H=\Delta_{\mathrm{HL}} H,
$$

where $\Delta_{\mathrm{HL}}=-\left(d d^{*}+d^{*} d\right)$ denotes the Hodge-Laplace operator.

The flow (1-9)-(1-10) can be interpreted as the connection Ricci flow [Streets 2008]. If we replace $H=d B$ by $F=d A$, i.e., replace a two-form by a one-form, then the flow (1-6)-(1-7) or (1-9)-(1-10) is exactly the Ricci Yang-Mills flow studied by Streets [2007] and Young [2008].

Ricci flow coupled with a one-form and a two-form. There is another generalized Ricci flow which connects to Thurston's conjecture - roughly stating that a three-dimensional manifold with a given topology has a canonical decomposition into simple three-dimensional manifolds, each of which admits one, and only one, of eight homogeneous geometries: $\mathbb{S}^{3}$, the round three-sphere; $\mathbb{R}^{3}$, the Euclidean space; $\mathbb{H}^{3}$, the standard hyperbolic space; $\mathbb{S}^{2} \times \mathbb{R} ; \mathbb{U}^{2} \times \mathbb{R} ;$ Nil, the three-dimensional nilpotent Heisenberg group; $\widetilde{\mathrm{SL}}(2, \mathbb{R})$; Sol, the three -dimensional solvable Lie group. The proof of Thurston's conjecture can be found in [Cao and Zhu 2006; Kleiner and Lott 2008; Morgan and Tian 2007; Perelman 2002].

To better understanding Thurston's conjecture, Gegenberg and Kunstatter [2004] proposed a generalized flow by considering the modified $3 D$ stringy theory. This flow is the Ricci flow coupled with evolution equations for a one-form and a two-form. As in (1-5), we define an energy functional

$$
\mathscr{F}^{(2)}: \operatorname{Met}(M) \times \mathscr{A}^{1}(M) \times \mathscr{A}^{2}(M) \times C^{\infty}(M) \rightarrow \mathbb{R}
$$

by

$$
\mathscr{F}^{(2)}(g, A, B, f)=\int_{M}\left(R+|\nabla f|^{2}-\frac{1}{12}|H|^{2}-\frac{1}{2}|F|^{2}\right) e^{-f} d V_{g},
$$

where $H=d B$, and $F=d A$. In [He et al. 2008], the authors showed that the gradient flow of $\mathscr{F}^{(2)}$ satisfies

$$
\begin{aligned}
\frac{\partial g_{i j}}{\partial t} & =-2 R_{i j}-2 \nabla_{i} \nabla_{j} f+\frac{1}{2} H_{i}{ }^{k \ell} H_{j k \ell}+2 F_{i}{ }^{k} F_{j k}, \\
\frac{\partial A_{i}}{\partial t} & =2 \nabla_{j} F^{j}{ }_{i}-2 F^{j}{ }_{i} \nabla_{j} f, \\
\frac{\partial B_{i j}}{\partial t} & =3 \nabla_{k} H^{k}{ }_{i j}-3 H^{k}{ }_{i j} \nabla_{k} f, \\
\frac{\partial f}{\partial t} & =-R-\Delta f+\frac{1}{4}|H|^{2}+|F|^{2},
\end{aligned}
$$

and under a family of diffeomorphisms the system (1-15)-(1-18) is equivalent to

$$
\begin{aligned}
\frac{\partial g_{i j}}{\partial t} & =-2 R_{i j}+\frac{1}{2} H_{i}{ }^{k \ell} H_{j k \ell}+2 F_{i}{ }^{k} F_{j k}, \\
\frac{\partial A_{i}}{\partial t} & =2 \nabla_{j} F_{i}^{j} \\
\frac{\partial B_{i j}}{\partial t} & =3 \nabla_{k} H^{k}{ }_{i j},
\end{aligned}
$$




$$
\frac{\partial f}{\partial t}=-R-\Delta f+|\nabla f|^{2}+\frac{1}{4}|H|^{2}+|F|^{2} .
$$

Using again the adjoint operator $d^{*}$, we have

$$
\frac{\partial F}{\partial t}=\Delta_{\mathrm{HL}} F, \quad \frac{\partial H}{\partial t}=\Delta_{\mathrm{HL}} H .
$$

The flow (1-19)-(1-21) clearly contains the Ricci flow, the flow (1-9)-(1-10) or the connection Ricci flow, and the Ricci Yang-Mills flow; we expect this flow can give another proof of the Poincaré conjecture and Thurston's generalization conjecture, with less analysis on singularities.

Main results. For convenience, we refer to GRF the generalized Ricci flow and $\operatorname{RF}(A, B)$ the $\operatorname{Ricci}$ flow coupled with a one-form $A$ and a two-form $B$.

Let $(M, g)$ denote an $n$-dimensional closed Riemannian manifold with a three-form $H=\left\{H_{i j k}\right\}$. In the first part of this paper we consider the following GRF on $M$ :

$$
\begin{aligned}
\frac{\partial}{\partial t} g_{i j}(x, t) & =-2 R_{i j}(x, t)+\frac{1}{2} H_{i k \ell}(x, t) H_{j}^{k \ell}(x, t), \\
\frac{\partial}{\partial t} H(x, t) & =\Delta_{\mathrm{HL}, g(x, t)} H(x, t), \quad H(x, 0)=H(x), \quad g(x, 0)=g(x) .
\end{aligned}
$$

It is clearly from (1-9) and (1-13) that the gradient flow of the energy functional $\mathscr{F}^{(1)}$ is a special case of (1-24)-(1-25). The corresponding case that $H$ is closed is called the refined generalized Ricci flow (RGRF):

$$
\begin{aligned}
\frac{\partial}{\partial t} g_{i j}(x, t) & =-2 R_{i j}(x, t)+\frac{1}{2} H_{i k \ell}(x, t) H_{j}^{k \ell}(x, t), \\
\frac{\partial}{\partial t} H(x, t) & =-d d_{g(x, t)}^{*} H(x, t), \quad H(x, 0)=H(x), \quad g(x, 0)=g(x) .
\end{aligned}
$$

Here $d_{g(x, t)}^{*}$ is the dual operator of $d$ with respect to the metric $g(x, t)$.

Lemma 1.1. Under RGRF, $H(x, t)$ is closed if the initial value $H(x)$ is closed.

Proof. Since the exterior derivative $d$ is independent of the metric, we have

$$
\frac{\partial}{\partial t} d H(x, t)=d \frac{\partial}{\partial t} H(x, t)=d\left(-d d_{g(x, t)}^{*} H(x, t)\right)=0 .
$$

so $d H(x, t)=d H(x)=0$.

The closedness of $H$ is very important and has physical interpretation [Bakas 2007; Oliynyk et al. 2006]. Streets [2008] considered the connection Ricci flow in which $H$ is the geometric torsion of connection.

Proposition 1.2. If $(g(x, t), H(x, t))$ is a solution of RGRF and the initial value $H(x)$ is closed, then it is also a solution of GRF.

Proof. From Lemma 1.1 and the assumption we know that $H(x, t)$ are all closed. Hence

$$
\Delta_{\mathrm{HL}, g(x, t)} H(x, t)=-d d_{g(x, t)}^{*} H(x, t) .
$$


For GRF, a basic and natural question is the existence. The short-time existence for RGRF has been established in [He et al. 2008], where the authors have already showed the short-time existence for $\mathrm{RF}(A, B)$ obviously including RGRF. In this paper, we prove the short- time existence for RGF.

Theorem 1.3. There is a unique solution to GRF for a short time. More precisely, let $\left(M, g_{i j}(x)\right)$ be an $n$-dimensional closed Riemannian manifold with a three-form $H=\left\{H_{i j k}\right\}$, then there exists a constant $T=T(n)>0$ depending only on $n$ such that the evolution system

$$
\begin{gathered}
\frac{\partial}{\partial t} g_{i j}(x, t)=-2 R_{i j}(x, t)+\frac{1}{2} g^{k p}(x, t) g^{\ell q}(x, t) H_{i k \ell}(x, t) H_{j p q}(x, t), \\
\frac{\partial}{\partial t} H(x, t)=\Delta_{\mathrm{HL}, g(x, t)} H(x, t), \quad H(x, 0)=H(x), \quad g(x, 0)=g(x),
\end{gathered}
$$

has a unique solution $\left(g_{i j}(x, t), H_{i j k}(x, t)\right)$ for a short time $0 \leq t \leq T$.

After establishing the local existence, we are able to prove the higher derivatives estimates for GRF. Precisely, we have the following

Theorem 1.4. Suppose that $(g(x, t), H(x, t))$ is a solution to GRF on a closed manifold $M^{n}$ and $K$ is an arbitrary given positive constant. Then for each $\alpha>0$ and each integer $m \geq 1$ there exists a constant $C_{m}$ depending on $m, n, \max \{\alpha, 1\}$, and $K$ such that if

$$
|\operatorname{Rm}(x, t)|_{g(x, t)} \leq K, \quad|H(x)|_{g(x)} \leq K
$$

for all $x \in M$ and $t \in[0, \alpha / K]$, then

$$
\left|\nabla^{m-1} \operatorname{Rm}(x, t)\right|_{g(x, t)}+\left|\nabla^{m} H(x, t)\right|_{g(x, t)} \leq \frac{C_{m}}{t^{m / 2}}
$$

for all $x \in M$ and $t \in(0, \alpha / K]$.

As an application, we can prove the compactness theorem for GRF.

Theorem 1.5 (compactness for GRF). Let $\left\{\left(M_{k}, g_{k}(t), H_{k}(t), O_{k}\right)\right\}_{k \in \mathbb{N}}$ be a sequence of complete pointed solutions to GRF for $t \in[\alpha, \omega) \ni 0$ such that:

(i) There is a constant $C_{0}<\infty$ independent of $k$ such that

$$
\sup _{(x, t) \in M_{k} \times(\alpha, \omega)}\left|\operatorname{Rm}_{g_{k}(x, t)}\right|_{g_{k}(x, t)} \leq C_{0}, \quad \sup _{x \in M_{k}}\left|H_{k}(x, \alpha)\right|_{g_{k}(x, \alpha)} \leq C_{0} .
$$

(ii) There exists a constant $\iota_{0}>0$ satisfies

$$
\operatorname{inj}_{g_{k}(0)}\left(O_{k}\right) \geq \iota_{0} .
$$

Then there exists a subsequence $\left\{j_{k}\right\}_{k \in \mathbb{N}}$ such that

$$
\left(M_{j_{k}}, g_{j_{k}}(t), H_{j_{k}}(t), O_{j_{k}}\right) \rightarrow\left(M_{\infty}, g_{\infty}(t), H_{\infty}(t), O_{\infty}\right),
$$

converges to a complete pointed solution $\left(M_{\infty}, g_{\infty}(t), H_{\infty}(t), O_{\infty}\right), t \in[\alpha, \omega)$ to GRF as $k \rightarrow \infty$. 
In the second part of this paper, we consider the Ricci flow coupled with a one-form and a two-form. This flow is the gradient flow of $\mathscr{F}^{(2)}$ and takes the form

$$
\begin{aligned}
\frac{\partial}{\partial t} g_{i j}(x, t) & =-2 R_{i j}+\frac{1}{2} H_{i}{ }^{k \ell}(x, t) H_{j k \ell}(x, t)+2 F_{i}{ }^{k}(x, t) F_{j k}(x, t), \\
\frac{\partial}{\partial t} A_{i}(x, t) & =2 \nabla_{j} F^{j}{ }_{i}(x, t), \quad A_{i}(x, 0)=A_{i}(x), \quad g_{i j}(x, 0)=g_{i j}(x), \\
\frac{\partial}{\partial t} B_{i j}(x, t) & =3 \nabla_{k} H^{k}{ }_{i j}(x, t), \quad B_{i j}(x, 0)=B_{i j}(x) .
\end{aligned}
$$

Here $A=\left\{A_{i}\right\}$ and $B=\left\{B_{i j}\right\}$ is a one-form and a two-form on $M$, respectively, and $F=d A, H=d B$. For this flow, we can also prove the short-time existence, higher derivative estimates, and the compactness theorem.

The rest of this paper is organized as follows. In Section 2, we prove the short-time existence and uniqueness of the GRF for any given three-form $H$. In Section 3, we compute the evolution equations for the Levi-Civita connections, Riemann, Ricci, and scalar curvatures of a solution to the GRF. In Section 4, we establish higher derivative estimates for GRF, called Bernstein-Bando-Shi (BBS) derivative estimates (e.g., [Cao and Zhu 2006; Chow and Knopf 2004; Chow et al. 2007; 2008; 2010; Morgan and Tian 2007; Shi 1989]). In Section 5, we prove the compactness theorem for GRF by using BBS estimates. In Section 6, based on the work of [He et al. 2008], the similar results are established for $\operatorname{RF}(A, B)$.

\section{Short-time existence of GRF}

In this section we establish the short-time existence for GRF. Our method is standard: we use the DeTurck trick in Ricci flow to prove its short-time existence. We assume that $M$ is an $n$-dimensional closed Riemannian manifold with metric

$$
d \widetilde{s}^{2}=\widetilde{g}_{i j}(x) d x^{i} d x^{j}
$$

and with Riemannian curvature tensor $\left\{\widetilde{R}_{i j k \ell}\right\}$. We also assume that $\widetilde{H}=\left\{\widetilde{H}_{i j k}\right\}$ is a fixed three-form on $M$. In the following we put

$$
h_{i j}:=H_{i k \ell} H_{j}^{k \ell}
$$

Suppose the metrics

$$
d \widehat{s}_{t}^{2}=\frac{1}{2} \widehat{g}_{i j}(x, t) d x^{i} d x^{j}
$$

are the solutions of ${ }^{1}$

$$
\frac{\partial}{\partial t} \widehat{g}_{i j}(x, t)=-2 \widehat{R}_{i j}(x, t)+\widehat{h}_{i j}(x, t), \quad \hat{g}_{i j}(x, 0)=\widetilde{g}_{i j}(x)
$$

for a short time $0 \leq t \leq T$. Consider a family of smooth diffeomorphisms $\varphi_{t}: M \rightarrow M(0 \leq t \leq T)$ of $M$. Let

$$
d s_{t}^{2}:=\varphi_{t}^{*} d \widehat{s}_{t}^{2}, \quad 0 \leq t \leq T
$$

\footnotetext{
${ }^{1}$ In the following computations we don't need to use the evolution equation for $H(x, t)$, hence we only consider the evolution equation for metrics.
} 
be the pull-back metrics of $d \widehat{s}_{t}^{2}$. For coordinates system $x=\left\{x^{1}, \ldots, x^{n}\right\}$ on $M$, let

$$
d s_{t}^{2}=g_{i j}(x, t) d x^{i} d x^{j}
$$

and

$$
y(x, t)=\varphi_{t}(x)=\left\{y^{1}(x, t), \ldots, y^{n}(x, t)\right\} .
$$

Then we have

$$
g_{i j}(x, t)=\frac{\partial y^{\alpha}}{\partial x^{i}} \frac{\partial y^{\beta}}{\partial x^{j}} \widehat{g}_{\alpha \beta}(y, t) .
$$

By the assumption $\widehat{g}_{\alpha \beta}(x, t)$ are the solutions of

$$
\frac{\partial}{\partial t} \widehat{g}_{\alpha \beta}(x, t)=-2 \widehat{R}_{\alpha \beta}(x, t)+\widehat{h}_{\alpha \beta}(x, t), \quad \hat{g}_{\alpha \beta}(x, 0)=\widetilde{g}_{\alpha \beta}(x) .
$$

We use $R_{i j}, \widehat{R}_{i j}, \widetilde{R}_{i j} ; \Gamma_{i j}^{k}, \widehat{\Gamma}_{i j}^{k}, \widetilde{\Gamma}_{i j}^{k} ; \nabla, \hat{\nabla}, \tilde{\nabla} ; h_{i j}, \hat{h}_{i j}, \widetilde{h}_{i j}$ to denote the Ricci curvatures, Christoffel symbols, covariant derivatives, and products of the three-form $H$ with respect to $\tilde{g}_{i j}, \hat{g}_{i j}, g_{i j}$ respectively. Then

$$
\frac{\partial}{\partial t} g_{i j}(x, t)=\frac{\partial y^{\alpha}}{\partial x^{i}} \frac{\partial y^{\beta}}{\partial x^{j}}\left(\frac{\partial}{\partial t} \hat{g}_{\alpha \beta}(y, t)\right)+\frac{\partial}{\partial x^{i}}\left(\frac{\partial y^{\alpha}}{\partial t}\right) \frac{\partial y^{\beta}}{\partial x^{j}} \hat{g}_{\alpha \beta}(y, t)+\frac{\partial y^{\alpha}}{\partial x^{i}} \frac{\partial}{\partial x^{j}}\left(\frac{\partial y^{\beta}}{\partial t}\right) \hat{g}_{\alpha \beta}(y, t) .
$$

From (2-9) we have

$$
\frac{\partial}{\partial t} \widehat{g}_{\alpha \beta}(y, t)=-2 \widehat{R}_{\alpha \beta}(y, t)+\widehat{h}_{\alpha \beta}(y, t)+\frac{\partial \widehat{g}_{\alpha \beta}}{\partial y^{\gamma}} \frac{\partial y^{\gamma}}{\partial t},
$$

and

$$
\begin{aligned}
\frac{\partial}{\partial t} g_{i j}(x, t)=-2 \frac{\partial y^{\alpha}}{\partial x^{i}} & \frac{\partial y^{\beta}}{\partial x^{j}} \widehat{R}_{\alpha \beta}(y, t)+\frac{\partial y^{\alpha}}{\partial x^{i}} \frac{\partial y^{\beta}}{\partial x^{j}} \widehat{h}_{\alpha \beta}(y, t) \\
& +\frac{\partial y^{\alpha}}{\partial x^{i}} \frac{\partial y^{\beta}}{\partial x^{j}} \frac{\partial \hat{g}_{\alpha \beta}}{\partial y^{\gamma}} \frac{\partial y^{\gamma}}{\partial t}+\frac{\partial}{\partial x^{i}}\left(\frac{\partial y^{\alpha}}{\partial t}\right) \frac{\partial y^{\beta}}{\partial x^{j}} \widehat{g}_{\alpha \beta}(y, t)+\frac{\partial y^{\alpha}}{\partial x^{i}} \frac{\partial}{\partial x^{j}}\left(\frac{\partial y^{\beta}}{\partial t}\right) \widehat{g}_{\alpha \beta}(y, t) .
\end{aligned}
$$

Since

$$
R_{i j}(x, t)=\frac{\partial y^{\alpha}}{\partial x^{i}} \frac{\partial y^{\beta}}{\partial x^{j}} \hat{R}_{\alpha \beta}(y, t), \quad h_{i j}(x, t)=\frac{\partial y^{\alpha}}{\partial x^{i}} \frac{\partial y^{\beta}}{\partial x^{j}} \hat{h}_{\alpha \beta}(y, t),
$$

using [Shi 1989, §2, (29)], we obtain

$$
\frac{\partial}{\partial t} g_{i j}(x, t)=-2 R_{i j}(x, t)+h_{i j}(x, t)+\nabla_{i}\left(\frac{\partial y^{\alpha}}{\partial t} \frac{\partial x^{k}}{\partial y^{\alpha}} g_{j k}\right)+\nabla_{j}\left(\frac{\partial y^{\alpha}}{\partial t} \frac{\partial x^{k}}{\partial y^{\alpha}} g_{i k}\right) .
$$

According to DeTurck trick, we define $y(x, t)=\varphi_{t}(x)$ by the equation

$$
\frac{\partial y^{\alpha}}{\partial t}=\frac{\partial y^{\alpha}}{\partial x^{k}} g^{\beta \gamma}\left(\Gamma_{\beta \gamma}^{k}-\widetilde{\Gamma}_{\beta \gamma}^{k}\right), \quad y^{\alpha}(x, 0)=x^{\alpha},
$$

then (2-10) becomes

$$
\frac{\partial}{\partial t} g_{i j}(x, t)=-2 R_{i j}(x, t)+h_{i j}(x, t)+\nabla_{i} V_{j}+\nabla_{j} V_{i}, \quad g_{i j}(x, 0)=\tilde{g}_{i j}(x),
$$


where

$$
V_{i}=g_{i k} g^{\beta \gamma}\left(\Gamma_{\beta \gamma}^{k}-\widetilde{\Gamma}_{\beta \gamma}^{k}\right) .
$$

Lemma 2.1. The evolution equation (2-12) is a strictly parabolic system. Moreover,

$$
\begin{aligned}
& \frac{\partial}{\partial t} g_{i j}=g^{\alpha \beta} \widetilde{\nabla}_{\alpha} \widetilde{\nabla}_{\beta} g_{i j}-g^{\alpha \beta} g_{i p} \tilde{g}^{p q} \widetilde{R}_{j \alpha q \beta}-g^{\alpha \beta} g_{j p} \tilde{g}^{p q} \widetilde{R}_{i \alpha q \beta} \\
& \quad+\frac{1}{2} g^{\alpha \beta} g^{p q}\left(\tilde{\nabla}_{i} g_{p \alpha} \cdot \tilde{\nabla}_{j} g_{q \beta}+2 \tilde{\nabla}_{j p} \cdot \tilde{\nabla}_{q} g_{i \beta}-2 \tilde{\nabla}_{\alpha} g_{j p} \cdot \tilde{\nabla}_{\beta} g_{i q}-2 \tilde{\nabla}_{j} g_{p \alpha} \cdot \tilde{\nabla}_{\beta} g_{i q}-2 \widetilde{\nabla}_{i} g_{p \alpha} \cdot \tilde{\nabla}_{\beta} g_{j q}\right) \\
& +\frac{1}{2} g^{\alpha \beta} g^{p q} H_{i \alpha p} H_{j \beta q} .
\end{aligned}
$$

Proof. It is an immediate consequence of Lemma 2.1 of [Shi 1989].

Now we can prove the short-time existence of GRF.

Theorem 2.2. There is a unique solution to GRF for a short time. More precisely, let $\left(M, g_{i j}(x)\right)$ be an $n$-dimensional closed Riemannian manifold with a three-form $H=\left\{H_{i j k}\right\}$, then there exists a constant $T=T(n)>0$ depending only on $n$ such that the evolution system

$$
\begin{gathered}
\frac{\partial}{\partial t} g_{i j}(x, t)=-2 R_{i j}(x, t)+\frac{1}{2} g^{k p}(x, t) g^{\ell q}(x, t) H_{i k \ell}(x, t) H_{j p q}(x, t), \\
\frac{\partial}{\partial t} H(x, t)=\Delta_{\mathrm{HL}, g(x, t)} H(x, t), \quad H(x, 0)=H(x), \quad g(x, 0)=g(x),
\end{gathered}
$$

has a unique solution $\left(g_{i j}(x, t), H_{i j k}(x, t)\right)$ for a short time $0 \leq t \leq T$.

Proof. We proved that the first evolution equation is strictly parabolic by Lemma 2.1. Form the Ricci identity, we have $\Delta_{\mathrm{HL}, g(x, t)} H=\Delta_{\mathrm{LB}, g(x, t)} H+\mathrm{Rm} * H$ which is also strictly parabolic. Hence from the standard theory of parabolic systems, the evolution system has a unique solution.

\section{Evolution of curvatures}

The evolution equation for the Riemann curvature tensors to the usual Ricci flow (e.g., [Cao and Zhu 2006; Chow and Knopf 2004; Chow et al. 2007, 2008; 2010; Hamilton 1982; Morgan and Tian 2007; Shi 1989]) is given by

$$
\frac{\partial}{\partial t} R_{i j k \ell}=\Delta R_{i j k \ell}+\psi_{i j k \ell}
$$

where

$\psi_{i j k \ell}=2\left(B_{i j k \ell}-B_{i j \ell k}-B_{i \ell j k}+B_{i k j \ell}\right)-g^{p q}\left(R_{p j k \ell} R_{q \ell}+R_{i p k \ell} R_{q j}+R_{i j p \ell} R_{q k}+R_{i j k p} R_{q \ell}\right)$,

and $B_{i j k \ell}=g^{p r} g^{q s} R_{p i q j} R_{r k s \ell}$. From this we can easily deduce the evolution equation for the Riemann curvature tensors to GRF.

Let $v_{i j}(x, t)$ be any symmetric 2-tensor, we consider the flow

$$
\frac{\partial}{\partial t} g_{i j}(x, t)=v_{i j}(x, t) \text {. }
$$


Applying a formula in [Chow and Knopf 2004] to our case $v_{i j}:=-2 R_{i j}+\frac{1}{2} h_{i j}$ with $h_{i j}=H_{i k \ell} H_{j}{ }^{k \ell}$, we obtain

$$
\begin{aligned}
\frac{\partial}{\partial t} R_{i j k \ell}=- & \frac{1}{2}\left(-2 \nabla_{i} \nabla_{k} R_{j \ell}+\frac{1}{2} \nabla_{i} \nabla_{k} h_{j \ell}+2 \nabla_{i} \nabla_{\ell} R_{j k}-\frac{1}{2} \nabla_{i} \nabla_{\ell} h_{j k}\right. \\
& \left.\quad+2 \nabla_{j} \nabla_{k} R_{i \ell}-\frac{1}{2} \nabla_{j} \nabla_{k} h_{i \ell}-2 \nabla_{j} \nabla_{\ell} R_{i k}+\frac{1}{2} \nabla_{j} \nabla_{\ell} h_{i k}\right) \\
& \quad+\frac{1}{2} g^{p q}\left[R_{i j k p}\left(-2 R_{q \ell}+\frac{1}{2} h_{q \ell}\right)+R_{i j p \ell}\left(-2 R_{q k}+\frac{1}{2} h_{q k}\right)\right] \\
= & \nabla_{i} \nabla_{k} R_{j \ell}-\nabla_{i} \nabla_{\ell} R_{j k}-\nabla_{j} \nabla_{k} R_{i \ell}+\nabla_{j} \nabla_{\ell} R_{i k}-g^{p q}\left(R_{i j k p} R_{q \ell}+R_{i j p \ell} R_{q k}\right) \\
& \quad+\frac{1}{4}\left(-\nabla_{i} \nabla_{k} h_{j \ell}+\nabla_{i} \nabla_{\ell} h_{j k}+\nabla_{j} \nabla_{k} h_{i \ell}-\nabla_{j} \nabla_{\ell} h_{i k}\right)+\frac{1}{4} g^{p q}\left(R_{i j k p} h_{q \ell}+R_{i j p \ell} h_{q k}\right) \\
=\Delta & R_{i j k \ell}+2\left(B_{i j k \ell}-B_{i j \ell k}-B_{i l j k}+B_{i k j \ell}\right) \\
& \quad-g^{p q}\left(R_{p j k \ell} R_{q \ell}+R_{i p k \ell} R_{q j}+R_{i j p \ell} R_{q k}+R_{i j k p} R_{q \ell}\right) \\
& +\frac{1}{4}\left(-\nabla_{i} \nabla_{k} h_{j \ell}+\nabla_{i} \nabla_{\ell} h_{j k}+\nabla_{j} \nabla_{k} h_{i \ell}-\nabla_{j} \nabla_{\ell} h_{i k}\right)+\frac{1}{4} g^{p q}\left(R_{i j k p} h_{q \ell}+R_{i j p \ell} h_{q k}\right) .
\end{aligned}
$$

Proposition 3.1. For GRF we have

$$
\begin{aligned}
\frac{\partial}{\partial t} R_{i j k \ell}=\Delta R_{i j k \ell}+2\left(B_{i j k \ell}-B_{i j \ell k}-B_{i \ell j k}+B_{i k j \ell}\right) \\
\quad-g^{p q}\left(R_{p j k \ell} R_{q \ell}+R_{i p k \ell} R_{q j}+R_{i j p \ell} R_{q k}+R_{i j k p} R_{q \ell}\right) \\
\quad+\frac{1}{4}\left(-\nabla_{i} \nabla_{k} h_{j \ell}+\nabla_{i} \nabla_{\ell} h_{j k}+\nabla_{j} \nabla_{k} h_{i \ell}-\nabla_{j} \nabla_{\ell} h_{i k}\right)+\frac{1}{4} g{ }^{p q}\left(R_{i j k p} h_{q \ell}+R_{i j p \ell} h_{q k}\right) .
\end{aligned}
$$

In particular:

Corollary 3.2. For GRF we have

$$
\frac{\partial}{\partial t} \mathrm{Rm}=\Delta \mathrm{Rm}+\mathrm{Rm} * \mathrm{Rm}+H * H * \mathrm{Rm}+\sum_{i=0}^{2} \nabla^{i} H * \nabla^{2-i} H .
$$

Proof. From Proposition 3.1, we obtain

$$
\frac{\partial}{\partial t} \mathrm{Rm}=\Delta \mathrm{Rm}+\mathrm{Rm} * \mathrm{Rm}+\nabla^{2} h+h * \mathrm{Rm} .
$$

On the other hand, $h=H * H$ and

$$
\nabla^{2} h=\nabla(\nabla(H * H))=\nabla(\nabla H * H)=\nabla^{2} H * H+\nabla H * \nabla H .
$$

Combining these terms, we obtain the result.

Proposition 3.3. For GRF we have

$$
\begin{aligned}
\frac{\partial}{\partial t} R_{i k}=\Delta R_{i k}+2\left\langle R_{p i q k}, R_{p q}\right\rangle-2\left\langle R_{p i},\right. & \left.R_{p k}\right\rangle+\frac{1}{4}\left[\left\langle h_{\ell q}, R_{i \ell k q}\right\rangle+\left\langle R_{i p}, h_{k p}\right\rangle\right] \\
& +\frac{1}{4}\left[-\nabla_{i} \nabla_{k}|H|^{2}+g^{j \ell} \nabla_{i} \nabla_{\ell} h_{j k}+g^{j \ell} \nabla_{j} \nabla_{k} h_{i \ell}-\Delta h_{i k}\right] .
\end{aligned}
$$

Proof. Since

$$
\frac{\partial}{\partial t} R_{i k}=g^{j \ell} \frac{\partial}{\partial t} R_{i j k \ell}+2 g^{j p} g^{\ell q} R_{i j k \ell} R_{p q}
$$


and

$$
g^{i j} h_{i j}=g^{i j} H_{i p q} H_{j}^{p q}=g^{i j} g^{p r} g^{q s} H_{i p q} H_{j r s}=|H|^{2},
$$

it follows that

$$
\begin{aligned}
g^{j \ell}\left[-\nabla_{i} \nabla_{k} h_{j \ell}+\nabla_{i} \nabla_{\ell} h_{j k}+\nabla_{j} \nabla_{k} h_{i \ell}-\nabla_{j} \nabla_{\ell} h_{i k}+g^{p q} h_{q \ell} R_{i j k p}+g^{p q} h_{q k} R_{i j p \ell}\right] \\
\quad=-\nabla_{i} \nabla_{k}|H|^{2}+g^{j \ell} \nabla_{i} \nabla_{\ell} h_{j k}+g^{j \ell} \nabla_{j} \nabla_{k} h_{i \ell}-\Delta h_{i k}+g^{j \ell} g^{p q} h_{q \ell} R_{i j k p}+g^{p q} h_{q k} R_{i p} .
\end{aligned}
$$

From these identities, we get the result.

As a consequence, we obtain the evolution equation for scalar curvature.

Proposition 3.4. For GRF we have

$$
\frac{\partial}{\partial t} R=\Delta R+2|\operatorname{Ric}|^{2}-\frac{1}{2} \Delta|H|^{2}+\frac{1}{2}\left\langle h_{i j}, R_{i j}\right\rangle+\frac{1}{2} g^{i k} g^{j \ell} \nabla_{i} \nabla_{j} h_{k \ell} .
$$

Proof. From the usual evolution equation for scalar curvature under the Ricci flow, we have

$$
\begin{aligned}
\frac{\partial}{\partial t} R= & \Delta R+2|\operatorname{Ric}|^{2}+\frac{1}{4} g^{i k}\left[\left\langle h_{\ell q}, R_{i \ell k q}\right\rangle+\left\langle R_{i p}, h_{k p}\right\rangle\right] \\
& +\frac{1}{4} g^{i k}\left(-\nabla_{i} \nabla_{k}|H|^{2}+g^{j \ell} \nabla_{i} \nabla_{\ell} h_{j k}+g^{j \ell} \nabla_{j} \nabla_{k} h_{i \ell}-\Delta h_{i k}\right) \\
= & \Delta R+2|\operatorname{Ric}|^{2}+\frac{1}{4}\left\langle h_{i j}, R_{i j}\right\rangle+\frac{1}{4}\left\langle R_{i p}, h_{i p}\right\rangle \\
& -\frac{1}{4} \Delta|H|^{2}+\frac{1}{4} g^{i k} g^{j \ell} \nabla_{i} \nabla_{\ell} h_{j k}+\frac{1}{4} g^{i k} g^{j \ell} \nabla_{j} \nabla_{k} h_{i \ell}-\frac{1}{4} \Delta|H|^{2} .
\end{aligned}
$$

Simplifying the terms, we obtain the required result.

\section{Derivative estimates}

In this section we are going to prove BBS estimates. At first we review several basic identities of commutators $[\Delta, \nabla]$ and $[\partial / \partial t, \nabla]$. If $A=A(t)$ is a $t$-dependency tensor, and $\partial g_{i j} / \partial t=v_{i j}$, then applying the well-known formulas stated in [Chow and Knopf 2004] on GRF we have

$$
\begin{aligned}
\frac{\partial}{\partial t} \nabla \mathrm{Rm} & =\nabla \frac{\partial}{\partial t} \mathrm{Rm}+\mathrm{Rm} * \nabla(\mathrm{Rm}+H * H) \\
& =\nabla\left(\Delta \mathrm{Rm}+\mathrm{Rm} * \mathrm{Rm}+H * H * \mathrm{Rm}+\nabla^{2} H * H+\nabla H * \nabla H\right)+\mathrm{Rm} * \nabla \mathrm{Rm}+H * \nabla H * \mathrm{Rm} \\
& =\Delta(\nabla \mathrm{Rm})+\sum_{i+j=0} \nabla^{i} \mathrm{Rm} * \nabla^{j} \mathrm{Rm}+\sum_{i+j+k=0} \nabla^{i} H * \nabla^{j} H * \nabla^{k} \mathrm{Rm}+\sum_{i+j=0+2} \nabla^{i} H * \nabla^{j} H .
\end{aligned}
$$

More generally:

Proposition 4.1. For GRF and any nonnegative integer $\ell$ we have

$$
\frac{\partial}{\partial t} \nabla^{\ell} \mathrm{Rm}=\Delta\left(\nabla^{\ell} \mathrm{Rm}\right)+\sum_{i+j=\ell} \nabla^{i} \mathrm{Rm} * \nabla^{j} \mathrm{Rm}+\sum_{i+j+k=\ell} \nabla^{i} H * \nabla^{j} H * \nabla^{k} \mathrm{Rm}+\sum_{i+j=\ell+2} \nabla^{i} H * \nabla^{j} H .
$$


Proof. For $\ell=1$, this is (4-1). Suppose (4-2) holds for $1, \ldots, \ell$. By induction on $\ell$, for $\ell+1$ we have

$$
\begin{aligned}
\frac{\partial}{\partial t} & \nabla^{\ell+1} \mathrm{Rm} \\
& =\frac{\partial}{\partial t} \nabla\left(\nabla^{\ell} \mathrm{Rm}\right) \\
& =\nabla \frac{\partial}{\partial t}\left(\nabla^{\ell} \mathrm{Rm}\right)+\nabla^{\ell} \mathrm{Rm} * \nabla(\mathrm{Rm}+H * H) \\
& =\nabla\left[\Delta\left(\nabla^{\ell} \mathrm{Rm}\right)+\sum_{i+j=\ell} \nabla^{i} \mathrm{Rm} * \nabla^{j} \mathrm{Rm}+\sum_{i+j+k=\ell} \nabla^{i} H * \nabla^{j} H * \nabla^{k} \mathrm{Rm}+\sum_{i+j=\ell+2} \nabla^{i} H * \nabla^{j} H\right] \\
& =\Delta\left(\nabla^{\ell+1} \mathrm{Rm}\right)+\nabla \mathrm{Rm} * \nabla^{\ell} \mathrm{Rm}+\mathrm{Rm} * \nabla^{\ell+1} \mathrm{Rm} \nabla^{\ell} \mathrm{Rm} * \nabla \mathrm{Rm}+H * \nabla^{\ell} \mathrm{Rm}
\end{aligned}
$$

Simplifying these terms, we obtain the required result.

As an immediate consequence, we have an evolution inequality for $\left|\nabla^{l} \mathrm{Rm}\right|^{2}$.

Corollary 4.2. For GRF and any nonnegative integer $\ell$ we have

$$
\begin{aligned}
\frac{\partial}{\partial t}\left|\nabla^{\ell} \mathrm{Rm}\right|^{2} \leq \Delta\left|\nabla^{l} \mathrm{Rm}\right|^{2}-2\left|\nabla^{\ell+1} \mathrm{Rm}\right|^{2}+C \sum_{i+j=\ell}\left|\nabla^{i} \mathrm{Rm}\right| \cdot\left|\nabla^{j} \mathrm{Rm}\right| \cdot\left|\nabla^{\ell} \mathrm{Rm}\right| \\
+C \sum_{i+j+k=\ell}\left|\nabla^{i} H\right| \cdot\left|\nabla^{j} H\right| \cdot\left|\nabla^{k} \mathrm{Rm}\right| \cdot\left|\nabla^{\ell} \mathrm{Rm}\right|+C \sum_{i+j=\ell+2}\left|\nabla^{i} H\right| \cdot\left|\nabla^{j} H\right| \cdot\left|\nabla^{\ell} \mathrm{Rm}\right|,
\end{aligned}
$$

where $C$ represents universal constants depending only on the dimension of $M$.

Next we derive the evolution equations for the covariant derivatives of $H$.

Proposition 4.3. For GRF and any positive integer $\ell$ we have

$$
\frac{\partial}{\partial t} \nabla^{\ell} H=\Delta\left(\nabla^{\ell} H\right)+\sum_{i+j=\ell} \nabla^{i} H * \nabla^{j} \mathrm{Rm}+\sum_{i+j+k=\ell} \nabla^{i} H * \nabla^{j} H * \nabla^{k} H
$$

Proof. From the Bochner formula, the evolution equation for $H$ can be rewritten as

$$
\frac{\partial}{\partial t} H=\Delta H+\mathrm{Rm} * H .
$$


For $\ell=1$, we have

$$
\begin{aligned}
\frac{\partial}{\partial t} \nabla H & =\nabla \frac{\partial}{\partial t} H+H * \nabla(\mathrm{Rm}+H * H) \\
& =\nabla(\Delta H+\mathrm{Rm} * H)+H * \nabla \mathrm{Rm}+H * H * \nabla H \\
& =\nabla(\Delta H)+H * \nabla \mathrm{Rm}+\nabla H * \mathrm{Rm}+H * H * \nabla H \\
& =\Delta(\nabla H)+\nabla \mathrm{Rm} * H+\nabla H * \mathrm{Rm}+H * H * \nabla H .
\end{aligned}
$$

Using (4-2) and the same argument, we can prove the evolution equation for higher covariant derivatives.

Similarly, we have an evolution inequality for $\left|\nabla^{\ell} H\right|^{2}$.

Corollary 4.4. For GRF and for any positive integer $l$ we have

$$
\begin{aligned}
\frac{\partial}{\partial t}\left|\nabla^{l} H\right|^{2} \leq \Delta\left|\nabla^{\ell} H\right|^{2}-2\left|\nabla^{\ell+1} H\right|^{2} & \\
& +C \sum_{i+j=\ell}\left|\nabla^{i} H\right| \cdot\left|\nabla^{j} \mathrm{Rm}\right| \cdot\left|\nabla^{\ell} H\right|+C \sum_{i+j=\ell}\left|\nabla^{i} H\right| \cdot\left|\nabla^{j} H\right| \cdot\left|\nabla^{k} H\right| \cdot\left|\nabla^{l} H\right|,
\end{aligned}
$$

while

$$
\frac{\partial}{\partial t}|H|^{2} \leq \Delta|H|^{2}-2|\nabla H|^{2}+C \cdot|\mathrm{Rm}| \cdot|H|^{2} .
$$

Theorem 4.5. Suppose that $(g(x, t), H(x, t))$ is a solution to GRF on a closed manifold $M^{n}$ for a short time $0 \leq t \leq T$ and $K_{1}, K_{2}$ are arbitrary given nonnegative constants. Then there exists a constant $C_{n}$ depending only on $n$ such that if

$$
|\operatorname{Rm}(x, t)|_{g(x, t)} \leq K_{1}, \quad|H(x)|_{g(x)} \leq K_{2}
$$

for all $x \in M$ and $t \in[0, T]$, then

$$
|H(x, t)|_{g(x, t)} \leq K_{2} e^{C_{n} K_{1} t}
$$

for all $x \in M$ and $t \in[0, T]$.

Proof. Since

$$
\frac{\partial}{\partial t}|H|^{2} \leq \Delta|H|^{2}+C_{n}|\operatorname{Rm}| \cdot|H|^{2} \leq \Delta|H|^{2}+C_{n} K_{1}|H|^{2},
$$

using the maximum principle, we obtain $u(t) \leq u(0) e^{C_{n} K_{1} t}$, where $u(t)=|H|^{2}$.

The main result in this section is the following estimates for higher derivatives of Riemann curvature tensors and three-forms. Some special cases were proved in [Streets 2007; 2008; Young 2008].

Theorem 4.6. Suppose that $(g(x, t), H(x, t))$ is a solution to GRF on a compact manifold $M^{n}$ and $K$ is an arbitrary given positive constant. Then for each $\alpha>0$ and each integer $m \geq 1$ there exists a constant $C_{m}$ depending on $m, n, \max \{\alpha, 1\}$, and $K$ such that if

$$
|\operatorname{Rm}(x, t)|_{g(x, t)} \leq K, \quad|H(x)|_{g(x)} \leq K
$$


for all $x \in M$ and $t \in[0, \alpha / K]$, then

$$
\left|\nabla^{m-1} \operatorname{Rm}(x, t)\right|_{g(x, t)}+\left|\nabla^{m} H(x, t)\right|_{g(x, t)} \leq \frac{C_{m}}{t^{m / 2}}
$$

for all $x \in M$ and $t \in(0, \alpha / K]$.

Proof. In the following computations we always let $C$ be any constants depending on $n, m, \max \{\alpha, 1\}$, and $K$, which may take different values at different places. From the evolution equations and Theorem 4.5, we have

$$
\begin{aligned}
\frac{\partial}{\partial t}|\mathrm{Rm}|^{2} & \leq \Delta|\mathrm{Rm}|^{2}-2|\nabla \mathrm{Rm}|^{2}+C+C\left|\nabla^{2} H\right|+C|\nabla H|^{2}, \\
\frac{\partial}{\partial t}|H|^{2} & \leq \Delta|H|^{2}-2|\nabla H|^{2}+C, \\
\frac{\partial}{\partial t}|\nabla H|^{2} & \leq \Delta|\nabla H|^{2}-2\left|\nabla^{2} H\right|^{2}+C|\nabla \mathrm{Rm}| \cdot|\nabla H|+C|\nabla H|^{2} .
\end{aligned}
$$

Consider the function $u=t|\nabla H|^{2}+\gamma|H|^{2}+t|\mathrm{Rm}|^{2}$. Directly computing, we obtain

$$
\begin{aligned}
\frac{\partial}{\partial t} u & \leq \Delta u-2 t\left|\nabla^{2} H\right|^{2}+C t\left|\nabla^{2} H\right|+(C-2 \gamma)|\nabla H|^{2}+C+C \gamma-2 t|\nabla \mathrm{Rm}|^{2}+C t \cdot|\nabla \mathrm{Rm}| \cdot|\nabla H| \\
& \leq \Delta u+2(C-\gamma) \cdot|\nabla H|^{2}+C(1+\gamma) .
\end{aligned}
$$

If we choose $\gamma=C$, then $\frac{\partial}{\partial t} u \leq \Delta u+C$ which implies that $u \leq C e^{C t}$ since $u(0) \leq C$. With this estimate we are able to bound the first covariant derivative of $\mathrm{Rm}$ and the second covariant derivative of $H$. In order to control the term $|\nabla \mathrm{Rm}|^{2}$, we should use the evolution equations of $|H|^{2},|\nabla H|^{2}$ and $\left|\nabla^{2} H\right|^{2}$ to cancel with the bad terms, i.e., $\left|\nabla^{2} \mathrm{Rm}\right|^{2},\left|\nabla^{2} H\right|^{2}$, and $\left|\nabla^{3} H\right|^{2}$, in the evolution equation of $|\nabla \mathrm{Rm}|^{2}$ : $\frac{\partial}{\partial t}|\nabla \mathrm{Rm}|^{2}$

$\leq \Delta|\nabla \mathrm{Rm}|^{2}-2\left|\nabla^{2} \mathrm{Rm}\right|^{2}+C|\nabla \mathrm{Rm}|^{2}+\frac{C}{t^{1 / 2}}|\nabla \mathrm{Rm}|+C \cdot|\nabla \mathrm{Rm}| \cdot\left|\nabla^{3} H\right|+\frac{C}{t^{1 / 2}}\left|\nabla^{2} H\right| \cdot|\nabla \mathrm{Rm}|$, $\frac{\partial}{\partial t}\left|\nabla^{2} H\right|^{2} \leq \Delta\left|\nabla^{2} H\right|^{2}-2\left|\nabla^{3} H\right|^{2}+C \cdot\left|\nabla^{2} \mathrm{Rm}\right| \cdot\left|\nabla^{2} H\right|+\frac{C}{t^{1 / 2}}|\nabla \mathrm{Rm}| \cdot\left|\nabla^{2} H\right|+C\left|\nabla^{2} H\right|^{2}+\frac{C}{t}\left|\nabla^{2} H\right|$. As above, we define

$$
u:=t^{2}\left(\left|\nabla^{2} H\right|^{2}+|\nabla \mathrm{Rm}|^{2}\right)+t \beta\left(|\nabla H|^{2}+|\mathrm{Rm}|^{2}\right)+\gamma|H|^{2},
$$

and therefore, $\frac{\partial u}{\partial t} \leq \Delta u+C$. Motivated by cases for $m=1$ and $m=2$, for general $m$, we can define a function

$$
u:=t^{m}\left(\left|\nabla^{m} H\right|^{2}+\left|\nabla^{m-1} \mathrm{Rm}\right|^{2}\right)+\sum_{i=1}^{m-1} \beta_{i} t^{i}\left(\left|\nabla^{i} H\right|^{2}+\left|\nabla^{i-1} \mathrm{Rm}\right|^{2}\right)+\gamma|H|^{2}
$$

where $\beta_{i}$ and $\gamma$ are positive constants determined later. In the following, we always assume $m \geq 3$. 
Suppose that $\left|\nabla^{i-1} \mathrm{Rm}\right|+\left|\nabla^{i} H\right| \leq \frac{C_{i}}{t^{i / 2}}$, for $i=1,2, \ldots, m-1$. For such $i$, from Corollary 4.4, we have

$$
\begin{aligned}
\frac{\partial}{\partial t}\left|\nabla^{i} H\right|^{2} \leq \Delta\left|\nabla^{i} H\right|^{2}-2\left|\nabla^{i+1} H\right|^{2}+C \sum_{j=0}^{i}\left|\nabla^{j} H\right| \cdot\left|\nabla^{i-j} \operatorname{Rm}\right| \cdot\left|\nabla^{i} H\right| \\
\quad+C \sum_{j=0}^{i} \sum_{\ell=0}^{i-j}\left|\nabla^{j} H\right| \cdot\left|\nabla^{i-j-\ell} H\right| \cdot\left|\nabla^{\ell} H\right| \cdot\left|\nabla^{i} H\right| \\
\leq \Delta\left|\nabla^{i} H\right|^{2}-2\left|\nabla^{i+1} H\right|^{2}+C \cdot\left|\nabla^{i} H\right| \sum_{j=0}^{i} \frac{C_{j}}{t^{\frac{j}{2}}} \cdot \frac{C_{i-j+1}}{t^{\frac{i-j+1}{2}}}+C \cdot\left|\nabla^{i} H\right| \sum_{j=0}^{i} \sum_{\ell=0}^{i-j} \frac{C_{j}}{t^{\frac{j}{2}}} \cdot \frac{C_{i-j-\ell}}{t^{\frac{i-j-1}{2}}} \cdot \frac{C_{\ell}}{t^{\frac{l}{2}}} \\
\leq \Delta\left|\nabla^{i} H\right|^{2}-2\left|\nabla^{i+1} H\right|^{2}+\frac{C_{i}}{t^{\frac{i+1}{2}}}\left|\nabla^{i} H\right|+\frac{C_{i}}{t^{\frac{i}{2}}}\left|\nabla^{i} H\right| .
\end{aligned}
$$

Similarly, from Corollary 4.2 we also have

$$
\begin{aligned}
& \frac{\partial}{\partial t}\left|\nabla^{i-1} \mathrm{Rm}\right|^{2} \leq \Delta\left|\nabla^{i-1} \mathrm{Rm}\right|^{2}-2\left|\nabla^{i} \mathrm{Rm}\right|^{2}+C \sum_{j=0}^{i-1}\left|\nabla^{j} \mathrm{Rm}\right|\left|\nabla^{i-1-j} \mathrm{Rm}\right|\left|\nabla^{i-1} \mathrm{Rm}\right| \\
& +C \sum_{j=0}^{i-1} \sum_{\ell=0}^{i-1-j}\left|\nabla^{j} H\right| \cdot\left|\nabla^{i-1-j-\ell} H\right| \cdot\left|\nabla^{\ell} \mathrm{Rm}\right| \cdot\left|\nabla^{i-1} \mathrm{Rm}\right| \\
& +C \sum_{j=0}^{i+1}\left|\nabla^{j} H\right| \cdot\left|\nabla^{i+1-j} H\right| \cdot\left|\nabla^{i-1} \mathrm{Rm}\right| \\
& \leq \Delta\left|\nabla^{i-1} \mathrm{Rm}\right|^{2}-2\left|\nabla^{i} \mathrm{Rm}\right|^{2}+C \cdot\left|\nabla^{i-1} \mathrm{Rm}\right| \sum_{j=0}^{i-1} \frac{C_{j+1}}{t^{\frac{j+1}{2}}} \cdot \frac{C_{i-j}}{t^{\frac{i-j}{2}}} \\
& +C \cdot\left|\nabla^{i-1} \mathrm{Rm}\right| \sum_{j=0}^{i-1} \sum_{\ell=0}^{i-1-j} \frac{C_{j}}{t^{\frac{j}{2}}} \cdot \frac{C_{i-1-j-\ell}}{t^{\frac{i-1-j-\ell}{2}}} \cdot \frac{C_{\ell+1}}{t^{\frac{\ell+1}{2}}} \\
& +C \cdot\left|\nabla^{i-1} \mathrm{Rm}\right| \sum_{j=1}^{i} \frac{C_{j}}{t^{\frac{j}{2}}} \cdot \frac{C_{i+1-j}}{t^{\frac{i+1-j}{2}}}+C \cdot\left|\nabla^{i+1} H\right| \cdot \frac{C_{i}}{t^{\frac{i}{2}}} \\
& \leq \Delta\left|\nabla^{i-1} \mathrm{Rm}\right|^{2}-2\left|\nabla^{i} \mathrm{Rm}\right|^{2}+\frac{C_{i}}{t^{\frac{i+1}{2}}} \cdot\left|\nabla^{i-1} \mathrm{Rm}\right|+\frac{C_{i}}{t^{\frac{i}{2}}}\left|\nabla^{i+1} H\right|+\frac{C_{i}}{t^{\frac{i}{2}}}\left|\nabla^{i-1} \mathrm{Rm}\right| .
\end{aligned}
$$

The evolution inequality for $u$ is now given by

$$
\begin{aligned}
\frac{\partial u}{\partial t} \leq & m t^{m-1}\left(\left|\nabla^{m} H\right|^{2}+\left|\nabla^{m-1} \mathrm{Rm}\right|^{2}\right)+\sum_{i=1}^{m-1} i \beta_{i} t^{i-1}\left(\left|\nabla^{i} H\right|^{2}+\left|\nabla^{i-1} \mathrm{Rm}\right|^{2}\right) \\
& +t^{m}\left(\frac{\partial}{\partial t}\left|\nabla^{m} H\right|^{2}+\frac{\partial}{\partial t}\left|\nabla^{m-1} \mathrm{Rm}\right|^{2}\right)+\sum_{i=1}^{m-1} \beta_{i} t^{i}\left(\frac{\partial}{\partial t}\left|\nabla^{i} H\right|^{2}+\frac{\partial}{\partial t}\left|\nabla^{i-1} \mathrm{Rm}\right|^{2}\right)+\gamma \cdot \frac{\partial}{\partial t}|H|^{2} .
\end{aligned}
$$


It's easy to see that the second term is bounded by

$$
\sum_{i=1}^{m-1} i \beta_{i} t^{i-1} \frac{C_{i}}{t^{i}}=\sum_{i=1}^{m-1} i \beta_{i} C_{i} t^{-1}
$$

but this bound depends on $t$ and approaches to infinity when $t$ goes to zero. Hence we use the last second term to control this bad term. The evolution inequality for the third term is the combination of the inequalities

$$
\begin{aligned}
& \frac{\partial}{\partial t}\left|\nabla^{m} H\right|^{2} \\
& \begin{aligned}
\leq \Delta\left|\nabla^{m} H\right|^{2}-2\left|\nabla^{m+1} H\right|^{2}+C \sum_{i=0}^{m}\left|\nabla^{i} H\right| \cdot\left|\nabla^{m-i} \mathrm{Rm}\right| \cdot\left|\nabla^{m} H\right| \\
\quad+C \sum_{i=0}^{m} \sum_{j=0}^{m-i}\left|\nabla^{j} H\right| \cdot\left|\nabla^{m-i-j} H\right| \cdot\left|\nabla^{i} H\right| \cdot\left|\nabla^{m} H\right| \\
\leq \Delta\left|\nabla^{m} H\right|^{2}-2\left|\nabla^{m+1} H\right|^{2}+C\left|\nabla^{m} H\right|^{2}+C \cdot\left|\nabla^{m} \mathrm{Rm}\right| \cdot\left|\nabla^{m} H\right|+\frac{C_{m}}{t^{\frac{m+1}{2}}}\left|\nabla^{m} H\right|+\frac{C_{m}}{t^{\frac{m}{2}}}\left|\nabla^{m} H\right|
\end{aligned}
\end{aligned}
$$

and

$$
\begin{aligned}
\frac{\partial}{\partial t}\left|\nabla^{m-1} \mathrm{Rm}\right|^{2} \leq & \Delta\left|\nabla^{m-1} \mathrm{Rm}\right|^{2}-2\left|\nabla^{m} \mathrm{Rm}\right|^{2}+C \sum_{i=0}^{m-1}\left|\nabla^{i} \mathrm{Rm}\right| \cdot\left|\nabla^{m-1-i} \mathrm{Rm}\right| \cdot\left|\nabla^{m-1} \mathrm{Rm}\right| \\
& +C \sum_{i=0}^{m-1} \sum_{j=0}^{m-1-i}\left|\nabla^{j} H\right| \cdot\left|\nabla^{m-1-i-j} H\right| \cdot\left|\nabla^{i} \mathrm{Rm}\right| \cdot\left|\nabla^{m-1} \mathrm{Rm}\right| \\
& +C \sum_{i=0}^{m+1}\left|\nabla^{i} H\right| \cdot\left|\nabla^{m+1-i} H\right| \cdot\left|\nabla^{m-1} \mathrm{Rm}\right| \\
\leq & \Delta\left|\nabla^{m-1} \mathrm{Rm}\right|^{2}-2\left|\nabla^{m} \mathrm{Rm}\right|^{2}+C\left|\nabla^{m-1} \mathrm{Rm}\right|^{2}+\frac{C}{t^{\frac{1}{2}}} \cdot\left|\nabla^{m} H\right| \cdot\left|\nabla^{m-1} \mathrm{Rm}\right| \\
& +C\left|\nabla^{m+1} H\right|\left|\nabla^{m-1} \mathrm{Rm}\right|+\frac{C_{m}}{t^{\frac{m+1}{2}}}\left|\nabla^{m-1} \mathrm{Rm}\right|+\frac{C_{m}}{t^{\frac{m}{2}}}\left|\nabla^{m-1} \mathrm{Rm}\right| .
\end{aligned}
$$

Therefore we have

$$
\begin{aligned}
& \frac{\partial u}{\partial t} \leq m t^{m-1}\left(\left|\nabla^{m} H\right|^{2}+\left|\nabla^{m-1} \mathrm{Rm}\right|^{2}\right)+\sum_{i=1}^{m-1} i \beta_{i} t^{i-1}\left(\left|\nabla^{i} H\right|^{2}+\left|\nabla^{i-1} \mathrm{Rm}\right|^{2}\right) \\
&+t^{m}\left(\Delta\left|\nabla^{m} H\right|^{2}-2\left|\nabla^{m+1} H\right|^{2}+\frac{C}{t^{\frac{m+1}{2}}}\left|\nabla^{m} H\right|+C\left|\nabla^{m} H\right|^{2}\right. \\
&+C\left|\nabla^{m} \mathrm{Rm}\right| \cdot\left|\nabla^{m} H\right|+\Delta\left|\nabla^{m-1} \mathrm{Rm}\right|^{2} \\
&-2\left|\nabla^{m} \mathrm{Rm}\right|^{2}+\frac{C}{t^{\frac{m+1}{2}}}\left|\nabla^{m-1} \mathrm{Rm}\right|+C\left|\nabla^{m-1} \mathrm{Rm}\right|^{2} \\
&\left.+\frac{C}{t^{1 / 2}}\left|\nabla^{m} H\right| \cdot\left|\nabla^{m-1} \mathrm{Rm}\right|+C\left|\nabla^{m+1} H\right| \cdot\left|\nabla^{m-1} \mathrm{Rm}\right|\right)
\end{aligned}
$$




$$
\begin{aligned}
& +\sum_{i=1}^{m-1} \beta_{i} t^{i}\left(\frac{C_{i}}{t^{\frac{i+1}{2}}}\left|\nabla^{i-1} \mathrm{Rm}\right|+\Delta\left|\nabla^{i} H\right|^{2}-2\left|\nabla^{i+1} H\right|^{2}\right. \\
& \left.\quad+\Delta\left|\nabla^{i-1} \mathrm{Rm}\right|^{2}+\frac{C_{i}}{t^{\frac{i+1}{2}}}\left|\nabla^{i} H\right|+\frac{C_{i}}{t^{\frac{i}{2}}}\left|\nabla^{i+1} H\right|-2\left|\nabla^{i} \mathrm{Rm}\right|^{2}\right) \\
& +\gamma\left(\Delta|H|^{2}-2|\nabla H|^{2}+C\right) \\
& \leq \Delta u-2 t^{m}\left|\nabla^{m+1} H\right|^{2}+C t^{m}\left|\nabla^{m+1} H\right| \cdot\left|\nabla^{m-1} \mathrm{Rm}\right| \\
& -2 t^{m}\left|\nabla^{m} \mathrm{Rm}\right|^{2}+C t^{m}\left|\nabla^{m} \mathrm{Rm}\right| \cdot\left|\nabla^{m} H\right|+\sum_{i=0}^{m-2}(i+1) \beta_{i+1} t^{i}\left(\left|\nabla^{i+1} H\right|^{2}+\left|\nabla^{i} \mathrm{Rm}\right|^{2}\right) \\
& -2 \sum_{i=1}^{m-1} \beta_{i} t^{i}\left(\left|\nabla^{i+1} H\right|^{2}+\left|\nabla^{i} \mathrm{Rm}\right|^{2}\right)-2 \gamma|\nabla H|^{2}+\gamma C \\
& +C t^{m-1}\left|\nabla^{m} H\right|^{2}+C t^{m-1}\left|\nabla^{m-1} \mathrm{Rm}\right|^{2}+j t^{\frac{m-1}{2}}\left|\nabla^{m} H\right|+C t^{\frac{m-1}{2}}\left|\nabla^{m-1} \mathrm{Rm}^{m}\right| \\
& +C t^{m-\frac{1}{2}}\left|\nabla^{m} H\right| \cdot\left|\nabla^{m-1} \mathrm{Rm}^{m}+C t^{m}\right| \nabla^{m+1} H|\cdot| \nabla^{m-1} \mathrm{Rm} \mid \\
& +\sum_{i=1}^{m-1} \beta_{i} C_{i} t^{\frac{i}{2}}\left|\nabla^{i+1} H\right|+\sum_{i=1}^{m-1} \beta_{i} C_{i} t^{\frac{i-1}{2}}\left(\left|\nabla^{i} H\right|^{2}+\left|\nabla^{i-1} \mathrm{Rm}\right|\right) .
\end{aligned}
$$

Choosing

$$
(i+1) \beta_{i+1}=\beta_{i}, \quad \beta_{i}=\frac{A}{i !}, \quad i \geq 0,
$$

where $A$ is constant which is determined later, and noting that

$$
\sum_{i=1}^{m-1} \beta_{i} C_{i} t^{i / 2}\left|\nabla^{i+1} H\right| \leq \frac{1}{2} \sum_{i=1}^{m-1} \beta_{i} t^{i}\left|\nabla^{i+1} H\right|^{2}+\frac{1}{2} \sum_{i=1}^{m-1} \beta_{i} C_{i}^{2}
$$

and

$$
\begin{aligned}
\sum_{i=1}^{m-1} \beta_{i} C_{i} t^{\frac{i-1}{2}}\left(\left|\nabla^{i} H\right|+\left|\nabla^{i-1} \mathrm{Rm}\right|\right) & \\
& \leq \beta_{1} C_{1}(|\nabla H|+|\mathrm{Rm}|)+\sum_{i=1}^{m-2} \beta_{i+1} C_{i+1} t^{\frac{i}{2}}\left(\left|\nabla^{i+1} H\right|+\left|\nabla^{i} \mathrm{Rm}\right|\right) \\
& \leq \beta_{1} C_{1}(|\nabla H|+|\mathrm{Rm}|)+\sum_{i=1}^{m-2} \beta_{i+1} C_{i+1}\left(\frac{t^{i}\left|\nabla^{i+1} H\right|^{2}+t^{i}\left|\nabla^{i} \mathrm{Rm}\right|^{2}}{2 \beta_{i+1} C_{i+1} / \beta_{i}}+\frac{\beta_{i+1} C_{i+1}}{\beta_{i}}\right) \\
& \leq \beta_{1} C_{1}(|\nabla H|+|\mathrm{Rm}|)+\frac{1}{2} \sum_{i=1}^{m-2} \beta_{i} t^{i}\left(\left|\nabla^{i+1} H\right|^{2}+\left|\nabla^{i} \mathrm{Rm}\right|^{2}\right)+\sum_{i=1}^{m-2} \frac{\beta_{i+1}^{2} C_{i+1}^{2}}{\beta_{i}}
\end{aligned}
$$


we obtain

$$
\begin{aligned}
\frac{\partial}{\partial t} u \leq \Delta & u-2 t^{m}\left|\nabla^{m+1} H\right|^{2}+C t^{m}\left|\nabla^{m+1} H\right| \cdot\left|\nabla^{m-1} \mathrm{Rm}\right| \\
& -2 t^{m}\left|\nabla^{m} \mathrm{Rm}\right|^{2}+C t^{m}\left|\nabla^{m} H\right| \cdot\left|\nabla^{m} \mathrm{Rm}\right|+C t^{m-1}\left|\nabla^{m} H\right|^{2}+C t^{m-1}\left|\nabla^{m-1} \mathrm{Rm}\right|^{2} \\
& +C t^{m-\frac{1}{2}}\left|\nabla^{m} H\right| \cdot\left|\nabla^{m-1} \mathrm{Rm}\right|+\beta_{0}\left(|\nabla H|^{2}+|\mathrm{Rm}|^{2}\right) \\
& -\sum_{i=1}^{m-1} \beta_{i} t^{i}\left(\left|\nabla^{i+1} H\right|^{2}+\left|\nabla^{i} \mathrm{Rm}\right|^{2}\right)+\sum_{i=1}^{m-2} \beta_{i} t^{i}\left(\left|\nabla^{i+1} H\right|^{2}+\left|\nabla^{i} \mathrm{Rm}\right|^{2}\right) \\
& +\frac{1}{2} \beta_{m-1} t^{m-1}\left|\nabla^{m} H\right|^{2}+\beta_{1} C_{1}|\nabla H|-2 \gamma|\nabla H|^{2}+C+C \gamma \\
\leq \Delta & u+C t^{m-1}\left|\nabla^{m-1} \mathrm{Rm}\right|^{2}+C t^{m-1}\left|\nabla^{m} H\right|^{2} C t^{m-\frac{1}{2}}\left(\left|\nabla^{m} H\right|^{2}+\left|\nabla^{m-1} \mathrm{Rm}\right|^{2}\right)+\beta_{0}|\nabla H|^{2} \\
& +\beta_{1} C_{1}|\nabla H|-2 \gamma|\nabla H|^{2}+C+C \gamma-\frac{1}{2} \beta_{m-1} t^{m-1}\left|\nabla^{m} H\right|^{2}-\beta_{m-1} t^{m-1}\left|\nabla^{m} \mathrm{Rm}\right|^{2} \\
\leq \Delta & u \\
+ & \frac{1}{2}\left(C \sqrt{t}+C-\beta_{m-1}\right) t^{m-1}\left(\left.\left|\nabla^{m-1} \mathrm{Rm}^{2}+\right| \nabla^{m} H\right|^{2}\right) \\
& +\left(\beta_{0}+\beta_{1} C_{1}-2 \gamma\right)|\nabla H|^{2}+C+C \gamma+\beta_{1} C_{1} .
\end{aligned}
$$

When we chose $A$ and $\gamma$ sufficiently large, we obtain $\frac{\partial u}{\partial t} \leq \Delta u+C$, which implies that $u(t) \leq C$ since $u(0)$ is bounded.

Finally we give an estimate that plays a crucial role in the next section.

Corollary 4.7. Let $(g(x, t), H(x, t))$ be a solution of the generalized Ricci flow on a closed manifold $M$. If there are $\beta>0$ and $K>0$ such that

$$
|\operatorname{Rm}(x, t)|_{g(x, t)} \leq K, \quad|H(x)|_{g(x)} \leq K
$$

for all $x \in M$ and $t \in[0, T]$, where $T>\beta / K$, then there exists for each $m \in \mathbb{N}$ a constant $C_{m}$ depending on $m, n, \min \{\beta, 1\}$, and $K$ such that

$$
\left|\nabla^{m-1} \operatorname{Rm}(x, t)\right|_{g(x, t)}+\left|\nabla^{m} H(x, t)\right|_{g(x, t)} \leq C_{m} K^{m / 2}
$$

for all $x \in M$ and $t \in[\min \{\beta, 1\} / K, T]$.

Proof. The proof is the same as in [Chow et al. 2007]; we just copy it here. Let $\beta_{1}:=\min \{\beta, 1\}$. For any fixed point $t_{0} \in\left[\beta_{1} / K, T\right]$ we set $T_{0}:=t_{0}-\beta_{1} / K$. For $\bar{t}:=t-T_{0}$ we let $(\bar{g}(\bar{t}), \bar{H}(\bar{t}))$ be the solution of the system

$$
\frac{\partial \bar{g}}{\partial \bar{t}}=-2 \overline{\operatorname{Ric}}+\frac{1}{2} \bar{h}, \quad \frac{\partial \bar{H}}{\partial \bar{t}}=\Delta_{\mathrm{HL}, \bar{g}} \bar{H}, \quad \bar{g}(0)=g\left(T_{0}\right), \quad \bar{H}(0)=H\left(T_{0}\right) .
$$

The uniqueness of solution implies that $\bar{g}(\bar{t})=g\left(\bar{t}+T_{0}\right)=g(t)$ for $\bar{t} \in\left[0, \beta_{1} / K\right]$. By the assumption we have

$$
|\overline{\operatorname{Rm}}(x, \bar{t})|_{\bar{g}(x, \bar{t})} \leq K, \quad|\bar{H}(x)|_{\bar{g}(x)} \leq K
$$

for all $x \in M$ and $\bar{t} \in\left[0, \beta_{1} / K\right]$. Applying Theorem 4.5 with $\alpha=\beta_{1}$, we have

$$
\left|\bar{\nabla}^{m-1} \overline{\operatorname{Rm}}(x, \bar{t})\right|_{\bar{g}(x, \bar{t})}+\left|\bar{\nabla}^{m} H(x, \bar{t})\right|_{\bar{g}(x, \bar{t})} \leq \frac{\bar{C}_{m}}{\bar{t}^{m / 2}}
$$


for all $x \in M$ and $\bar{t} \in\left(0, \beta_{1} / K\right]$. We have $\bar{t}^{m / 2} \geq \beta_{1}^{m / 2} 2^{-m / 2} K^{-m / 2}$ if $\bar{t} \in\left[\beta_{1} / 2 K, \beta_{1} / K\right]$. Taking $\bar{t}=\beta_{1} / K$, we obtain

$$
\left|\nabla^{m-1} \operatorname{Rm}\left(x, t_{0}\right)\right|_{g\left(x, t_{0}\right)}+\left|\nabla^{m} H\left(x, t_{0}\right)\right|_{g\left(x, t_{0}\right)} \leq \frac{2^{m / 2} \bar{C}_{m} K^{m / 2}}{\beta_{1}^{m / 2}}
$$

for all $x \in M$. Since $t_{0} \in[\beta / K, T]$ was arbitrary, the result follows.

\section{Compactness theorem}

In this section we prove the compactness theorem for our generalized Ricci flow. We follow [Hamilton 1995] on the compactness theorem for the usual Ricci flow.

We review several definitions from [Chow et al. 2007]. Throughout this section, all Riemannian manifolds are smooth manifolds of dimensions $n$. The covariant derivative with respect to a metric $g$ will be denoted by ${ }^{g} \nabla$.

Definition 5.1. Let $K \subset M$ be a compact set and let $\left\{g_{k}\right\}_{k \in \mathbb{N}}, g_{\infty}$, and $g$ be Riemannian metrics on $M$. For $p \in\{0\} \cup \mathbb{N}$ we say that $g_{k}$ converges in $C^{p}$ to $g_{\infty}$ uniformly on $K$ with respect to $g$ if for every $\epsilon>0$ there exists $k_{0}=k_{0}(\epsilon)>0$ such that for $k \geq k_{0}$,

$$
\left\|g_{k}-g_{\infty}\right\|_{C^{p} ; K, g}:=\left.\left.\sup _{0 \leq \alpha \leq p} \sup _{x \in K}\right|^{g} \nabla^{\alpha}\left(g_{k}-g_{\infty}\right)(x)\right|_{g}<\epsilon .
$$

Since we consider a compact set, the choice of background metric $g$ does not change the convergence. Hence we may choose $g=g_{\infty}$.

Definition 5.2. Suppose $\left\{U_{k}\right\}_{k \in \mathbb{N}}$ is an exhaustion ${ }^{2}$ of a smooth manifold $M$ by open sets and $g_{k}$ are Riemannian metrics on $U_{k}$. We say that $\left(U_{k}, g_{k}\right)$ converges in $C^{\infty}$ to $\left(M, g_{\infty}\right)$ uniformly on compact sets in $M$ if for any compact set $K \subset M$ and any $p>0$ there exists $k_{0}=k_{0}(K, p)$ such that $\left\{g_{k}\right\}_{k \geq k_{0}}$ converges in $C^{p}$ to $g_{\infty}$ uniformly on $K$.

A pointed Riemannian manifold is a 3-tuple $(M, g, O)$, where $(M, g)$ is a Riemannian manifold and $O \in M$ is a basepoint. If the metric $g$ is complete, the 3-tuple is called a complete pointed Riemannian manifold. We say $(M, g(t), H(t), O), t \in(\alpha, \omega)$, is a pointed solution to the generalized Ricci flow if $(M, g(t), H(t))$ is a solution to the generalized Ricci flow.

The so-called Cheeger-Gromov convergence in $C^{\infty}$ is defined as follows:

Definition 5.3. A given sequence $\left\{\left(M_{k}, g_{k}, O_{k}\right)\right\}_{k \in \mathbb{N}}$ of complete pointed Riemannian manifolds converges to a complete pointed Riemannian manifold $\left(M_{\infty}, g_{\infty}, O_{\infty}\right)$ if there exist

(i) an exhaustion $\left\{U_{k}\right\}_{k \in \mathbb{N}}$ of $M_{\infty}$ by open sets with $O_{\infty} \in U_{k}$, and

(ii) a sequence of diffeomorphisms $\Phi_{k}: M_{\infty} \ni U_{k} \rightarrow V_{k}:=\Phi_{k}\left(U_{k}\right) \subset M_{k}$ with $\Phi_{k}\left(O_{\infty}\right)=O_{k}$ such that $\left(U_{k}, \Phi_{k}^{*}\left(\left.g_{k}\right|_{V_{k}}\right)\right)$ converges in $C^{\infty}$ to $\left(M_{\infty}, g_{\infty}\right)$ uniformly on compact sets in $M_{\infty}$.

\footnotetext{
${ }^{2}$ If for any compact set $K \subset M$ there exists $k_{0} \in \mathbb{N}$ such that $U_{k} \supset K$ for all $k \geq k_{0}$
} 
The corresponding convergence for the generalized Ricci flow is similar to the convergence for the usual Ricci flow introduced by Hamilton [1995].

Definition 5.4. A given sequence $\left\{\left(M_{k}, g_{k}(t), H_{k}(t), O_{k}\right)\right\}_{k \in \mathbb{N}}$ of complete pointed solutions to the GRF converges to a complete pointed solution to the GRF

$$
\left(M_{\infty}, g_{\infty}(t), H_{\infty}(t), O_{\infty}\right), \quad t \in(\alpha, \omega),
$$

if there exist

(i) an exhaustion $\left\{U_{k}\right\}_{k \in \mathbb{N}}$ of $M_{\infty}$ by open sets with $O_{\infty} \in U_{k}$,

(ii) a sequence of diffeomorphisms $\Phi_{k}: M_{\infty} \ni U_{k} \rightarrow V_{k}:=\Phi_{k}\left(U_{k}\right) \subset M_{k}$ with $\Phi_{k}\left(O_{\infty}\right)=O_{k}$ such that $\left(U_{k} \times(\alpha, \omega), \Phi_{k}^{*}\left(\left.g_{k}(t)\right|_{V_{k}}\right)+d t^{2}, \Phi_{k}^{*}\left(\left.H_{k}(t)\right|_{V_{k}}\right)\right)$ converges in $C^{\infty}$ to

$$
\left(M_{\infty} \times(\alpha, \omega), g_{\infty}(t)+d t^{2}, H_{\infty}(t)\right)
$$

uniformly on compact sets in $M_{\infty} \times(\alpha, \omega)$. Here we denote by $d t^{2}$ the standard metric on $(\alpha, \omega)$.

Let $\operatorname{inj}_{g}(O)$ be the injectivity radius of the metric $g$ at the point $O$. The following compactness theorem is due to Cheeger and Gromov.

Theorem 5.5 (compactness for metrics). Let $\left\{\left(M_{k}, g_{k}, O_{k}\right)\right\}_{k \in \mathbb{N}}$ be a sequence of complete pointed Riemannian manifolds satisfying these conditions:

(i) For all $p \geq 0$ and $k \in \mathbb{N}$, there is a sequence of constants $C_{p}<\infty$ independent of $k$ such that

$$
\left.\left.\right|^{g_{k}} \nabla^{p} \operatorname{Rm}\left(g_{k}\right)\right|_{g_{k}} \leq C_{p}
$$

on $M_{k}$.

(ii) There exists some constant $\iota_{0}>0$ such that

$$
\operatorname{inj}_{g_{k}}\left(O_{k}\right) \geq \iota_{0}
$$

for all $k \in \mathbb{N}$.

Then there exists a subsequence $\left\{j_{k}\right\}_{k \in \mathbb{N}}$ such that $\left\{\left(M_{j_{k}}, g_{j_{k}}, O_{j_{k}}\right)\right\}_{k \in \mathbb{N}}$ converges to a complete pointed Riemannaian manifold $\left(M_{\infty}^{n}, g_{\infty}, O_{\infty}\right)$ as $k \rightarrow \infty$.

As a consequence of Theorem 5.5, we state our compactness theorem for GRF.

Theorem 5.6 (compactness for GRF). Let $\left\{\left(M_{k}, g_{k}(t), H_{k}(t), O_{k}\right)\right\}_{k \in \mathbb{N}}$ be a sequence of complete pointed solutions to GRF for $t \in[\alpha, \omega) \ni 0$ satisfying these conditions:

(i) There is a constant $C_{0}<\infty$ independent of $k$ such that

$$
\sup _{(x, t) \in M_{k} \times(\alpha, \omega)}\left|\operatorname{Rm}\left(g_{k}(x, t)\right)\right|_{g_{k}(x, t)} \leq C_{0}, \quad \sup _{x \in M_{k}}\left|H_{k}(x, \alpha)\right|_{g_{k}(x, \alpha)} \leq C_{0} .
$$

(ii) There exists a constant $\iota_{0}>0$ satisfying

$$
\operatorname{inj}_{g_{k}(0)}\left(O_{k}\right) \geq \iota_{0}
$$


Then there exists a subsequence $\left\{j_{k}\right\}_{k \in \mathbb{N}}$ such that

$$
\left(M_{j_{k}}, g_{j_{k}}(t), H_{j_{k}}(t), O_{j_{k}}\right) \rightarrow\left(M_{\infty}, g_{\infty}(t), H_{\infty}(t), O_{\infty}\right)
$$

converges to a complete pointed solution $\left(M_{\infty}, g_{\infty}(t), H_{\infty}(t), O_{\infty}\right), t \in[\alpha, \omega)$, to GRF as $k \rightarrow \infty$.

To prove Theorem 5.6 we extend a lemma for Ricci flow to GRF. After establishing this lemma, the proof of Theorem 5.6 is similar to that of Theorem 3.10 in [Chow et al. 2007].

Lemma 5.7. Let $(M, g)$ be a Riemannian manifold with a background metric $g$, let $K$ be a compact subset of $M$, and let $\left(g_{k}(x, t), H_{k}(x, t)\right)$ be a collection of solutions to the generalized Ricci flow defined on neighborhoods of $K \times[\beta, \psi]$, where $t_{0} \in[\beta, \psi]$ is a fixed time. Suppose that:

(i) The metrics $g_{k}\left(x, t_{0}\right)$ are all uniformly equivalent to $g(x)$ on $K$, i.e., for all $V \in T_{x} M, k$, and $x \in K$,

$$
C^{-1} g(x)(V, V) \leq g_{k}\left(x, t_{0}\right)(V, V) \leq C g(x)(V, V),
$$

where $C<\infty$ is a constant independent of $V, k$, and $x$.

(ii) The covariant derivatives of the metrics $g_{k}\left(x, t_{0}\right)$ with respect to the metric $g(x)$ are all uniformly bounded on $K$, i.e., for all $k$ and $p \geq 1$,

$$
\left|{ }^{g} \nabla^{p} g_{k}\left(x, t_{0}\right)\right|_{g(x)}+\left.\left.\right|^{g} \nabla^{p-1} H_{k}\left(x, t_{0}\right)\right|_{g(x)} \leq C_{p}
$$

where $C_{p}<\infty$ is a sequence of constants independent of $k$.

(iii) The covariant derivatives of the curvature tensors $\operatorname{Rm}\left(g_{k}(x, t)\right)$ and of the forms $H_{k}(x, t)$ are uniformly bounded with respect to the metric $g_{k}(x, t)$ on $K \times[\beta, \psi]$, i.e., for all $k$ and $p \geq 0$,

$$
\left.\left.\right|^{g_{k}} \nabla^{p} \operatorname{Rm}\left(g_{k}(x, t)\right)\right|_{g_{k}(x, t)}+\left.\left.\right|^{g_{k}} \nabla^{p} H_{k}(x, t)\right|_{g_{k}(x, t)} \leq C_{p}^{\prime}
$$

where $C_{p}^{\prime}$ is a sequence of constants independent of $k$.

Then the metrics $g_{k}(x, t)$ are uniformly equivalent to $g(x)$ on $K \times[\beta, \psi]$, i.e.,

$$
B\left(t, t_{0}\right)^{-1} g(x)(V, V) \leq g_{k}(x, t)(V, V) \leq B\left(t, t_{0}\right) g(x)(V, V),
$$

where $B\left(t, t_{0}\right)=C e^{C_{0}^{\prime}\left|t-t_{0}\right|}$ (here the constant $C_{0}^{\prime}$ may not be equal to the previous one), and the timederivatives and covariant derivatives of the metrics $g_{k}(x, t)$ with respect to the metric $g(x)$ are uniformly bounded on $K \times[\beta, \psi]$, i.e., for each $(p, q)$ there is a constant $\widetilde{C}_{p, q}$ independent of $k$ such that

$$
\left|\frac{\partial^{q}}{\partial t^{q}} g^{g} \nabla^{p} g_{k}(x, t)\right|_{g(x)}+\left|\frac{\partial^{q}}{\partial t^{q}} g^{g} \nabla^{p-1} H_{k}(x, t)\right|_{g(x)} \leq \widetilde{C}_{p, q}
$$

for all $k$.

Proof. We use [Chow et al. 2007, Lemma 3.13]: Suppose that the metrics $g_{1}$ and $g_{2}$ are equivalent, i.e., $C^{-1} g_{1} \leq g_{2} \leq C g_{1}$. Then for any $(p, q)$-tensor $T$ we have $|T|_{g_{2}} \leq C^{(p+q) / 2}|T|_{g_{1}}$. We denote by $h$ 
the tensor $h_{i j}:=g^{k p} g^{l q} H_{i k l} H_{j p q}$. In the following we denote by $C$ a constant depending only on $n, \beta$, and $\psi$, which may take different values at different places. For any tangent vector $V \in T_{x} M$ we have

$$
\frac{\partial}{\partial t} g_{k}(x, t)(V, V)=-2 \operatorname{Ric}\left(g_{k}(x, t)\right)(V, V)+\frac{1}{2} h_{k}(x, t)(V, V),
$$

and therefore

$$
\begin{aligned}
\left|\frac{\partial}{\partial t} \log g_{k}(x, t)(V, V)\right| & =\left|\frac{-2 \operatorname{Ric}\left(g_{k}(x, t)\right)(V, V)+\frac{1}{2} h_{k}(x, t)(V, V)}{g_{k}(x, t)(V, V)}\right| \\
& \leq C_{0}^{\prime}+C\left|H_{k}(x, t)\right|_{g_{k}(x, t)}^{2} \\
& \leq C_{0}^{\prime}+C C_{0}^{\prime 2}=: \bar{C}
\end{aligned}
$$

since

$$
\left|\operatorname{Ric}\left(g_{k}(x, t)\right)(V, V)\right| \leq C_{0}^{\prime} g_{k}(x, t)(V, V), \quad\left|h_{k}(x, t)(V, V)\right| \leq C\left|H_{k}(x, t)\right|_{g_{k}(x, t)}^{2} g_{k}(x, t)(V, V) .
$$

Integrating on both sides, we have

$$
\bar{C}\left|t_{1}-t_{0}\right| \geq \int_{t_{0}}^{t_{1}}\left|\frac{\partial}{\partial t} \log g_{k}(x, t)(V, V)\right| d t \geq\left|\int_{t_{0}}^{t_{1}} \frac{\partial}{\partial t} \log g_{k}(t)(V, V) d t\right|=\left|\log \frac{g_{k}\left(x, t_{1}\right)(V, V)}{g_{k}\left(x, t_{0}\right)(V, V)}\right|,
$$

and hence we conclude that

$$
e^{-\bar{C}\left|t_{1}-t_{0}\right|} g_{k}\left(x, t_{0}\right)(V, V) \leq g_{k}\left(x, t_{1}\right)(V, V) \leq e^{\bar{C}\left|t_{1}-t_{0}\right|} g_{k}\left(x, t_{0}\right)(V, V) .
$$

From the assumption (i), it immediately deduces from above that

$$
C^{-1} e^{-\bar{C}\left|t_{1}-t_{0}\right|} g(x)(V, V) \leq g_{k}\left(x, t_{1}\right)(V, V) \leq C e^{\bar{C}\left|t_{1}-t_{0}\right|} g(x)(V, V) .
$$

Since $t_{1}$ was arbitrary, the first part is proved. From the definition (or see [Chow et al. 2007, p. 134, (37)]), we have

$$
\left(g_{k}\right)^{e c}\left({ }^{g} \nabla_{a}\left(g_{k}\right)_{b c}+{ }^{g} \nabla_{b}\left(g_{k}\right)_{a c}-{ }^{g} \nabla_{c}\left(g_{k}\right)_{a b}\right)=2\left({ }^{g_{k}} \Gamma\right)_{a b}^{e}-2\left({ }^{g} \Gamma\right)_{a b}^{e} .
$$

Thus $\left.\right|^{g_{k}} \Gamma(x, t)-\left.{ }^{g} \Gamma(x)\right|_{g(x)} \leq\left.\left. C\right|^{g} \nabla g_{k}(x, t)\right|_{g_{k}(x)}$. On the other hand,

$$
{ }^{g} \nabla_{a}\left(g_{k}\right)_{b c}=\left(g_{k}\right)_{e b}\left[\left({ }^{g_{k}} \Gamma\right)_{a c}^{e}-\left({ }^{g} \Gamma\right)_{a c}^{e}\right]+\left(g_{k}\right)_{e c}\left[\left({ }^{g_{k}} \Gamma\right)_{a b}^{e}-\left({ }^{g} \Gamma\right)_{a b}^{e}\right],
$$

it follows that $\left.\left.\right|^{g} \nabla g_{k}(x, t)\right|_{g_{k}(x, t)} \leq\left. C\right|^{g_{k}} \Gamma(x, t)-\left.{ }^{g} \Gamma(x)\right|_{g_{k}(x, t)}$ and therefore

$$
{ }^{g} \nabla g_{k} \text { is equivalent to }{ }^{g_{k}} \Gamma-{ }^{g} \Gamma={ }^{g_{k}} \nabla-{ }^{g} \nabla .
$$

The evolution equation for ${ }^{g} \Gamma$ is

$$
\begin{aligned}
\frac{\partial}{\partial t}\left({ }^{g_{k}} \Gamma\right)_{a b}^{c}=-( & \left.g_{k}\right)^{c d}\left[\left({ }^{g_{k}} \nabla\right)_{a}\left(\operatorname{Ric}\left(g_{k}\right)\right)_{b d}+\left({ }^{g_{k}} \nabla\right)_{b}\left(\operatorname{Ric}\left(g_{k}\right)\right)_{a d}\right. \\
& \left.-\left({ }^{g_{k}} \nabla\right)_{d}\left(\operatorname{Ric}\left(g_{k}\right)\right)_{a b}\right]+\frac{1}{4}\left(g_{k}\right)^{c d}\left[\left({ }^{g_{k}} \nabla\right)_{a}\left(h_{k}\right)_{b d}+\left({ }^{g_{k}} \nabla\right)_{b}\left(h_{k}\right)_{a d}-\left({ }^{g_{k}} \nabla\right)_{d}\left(h_{k}\right)_{a b}\right] .
\end{aligned}
$$


Since ${ }^{g} \Gamma$ does not depend on $t$, it follows from the assumptions that

$$
\begin{aligned}
\left|\frac{\partial}{\partial t}\left({ }^{g_{k}} \Gamma-{ }^{g} \Gamma\right)\right|_{g_{k}} & \leq\left.\left. C\right|^{g_{k}} \nabla\left(\operatorname{Ric}\left(g_{k}\right)\right)\right|_{g_{k}}+\left.\left.C\right|^{g_{k}} \nabla\left(h_{k}\right)\right|_{g_{k}} \\
& \leq C C_{1}^{\prime}+\left.\left.C\right|^{g_{k}} \nabla H_{k}\right|_{g_{k}} \cdot\left|H_{k}\right|_{g_{k}} \leq C_{1}^{\prime} .
\end{aligned}
$$

Integrating on both sides,

$$
C_{1}^{\prime}\left|t_{1}-t_{0}\right| \geq\left|\int_{t_{0}}^{t_{1}} \frac{\partial}{\partial t}\left(g^{k} \Gamma(t)-{ }^{g} \Gamma\right) d t\right|_{g_{k}} \geq\left.\right|^{g_{k}} \Gamma\left(t_{1}\right)-{ }^{g} \Gamma\left|g_{k}-\right|^{g_{k}} \Gamma\left(t_{0}\right)-{ }^{g} \Gamma \mid g_{k} .
$$

Hence we obtain

$$
\begin{aligned}
\left.\right|^{g_{k}} \Gamma(t)-{ }^{g} \Gamma \mid g_{k} & \leq C_{1}^{\prime}\left|t_{1}-t_{0}\right|+\left.\right|^{g_{k}} \Gamma\left(t_{0}\right)-{ }^{g} \Gamma \mid g_{k} \\
& \leq C_{1}^{\prime}\left|t_{1}-t_{0}\right|+\left.\left.C\right|^{g} \nabla g_{k}\left(t_{0}\right)\right|_{g_{k}} \\
& \leq C_{1}^{\prime}\left|t-t_{0}\right|+\left.C\right|^{g} \nabla g_{k}\left(t_{0}\right) \mid g \\
& \leq C_{1}^{\prime}\left|t-t_{0}\right|+C_{1} .
\end{aligned}
$$

The equivalency of metrics tells us that

$$
\begin{aligned}
\left.\left.\right|^{g} \nabla g_{k}(t)\right|_{g} & \leq\left. B\left(t, t_{0}\right)^{3 / 2}\right|^{g} \nabla g_{k}(t)\left|g_{k} \leq B\left(t, t_{0}\right)^{3 / 2} \cdot C\right|^{g_{k}} \Gamma(t)-{ }^{g} \Gamma \mid g_{k} \\
& \leq B\left(t, t_{0}\right)^{3 / 2}\left(C_{1}^{\prime}\left|t-t_{0}\right|+C^{\prime}\right) .
\end{aligned}
$$

Since $\left|t-t_{0}\right| \leq \psi-\beta$, it follows that $\left.\left.\right|^{g} \nabla g_{k}(t)\right|_{g} \leq \widetilde{C}_{1,0}$ for some constant $\widetilde{C}_{1,0}$. But $g$ and $g_{k}$ are equivalent, we have

$$
\left|H_{k}(t)\right|_{g} \leq C\left|H_{k}(t)\right|_{g_{k}} \leq C C_{1}^{\prime}=\widetilde{C}_{1,0} .
$$

From the assumptions, we also have

$$
\begin{aligned}
\left.\left.\right|^{g} \nabla H_{k}\right|_{g} & \leq\left|\left(^{g} \nabla-{ }^{g_{k}} \nabla\right) H_{k}+{ }^{g_{k}} \nabla H_{k}\right|_{g} \\
& \leq\left.\left. C\right|^{g} \nabla g_{k}\right|_{g} \cdot\left|H_{k}\right|_{g}+\left.\left.C\right|^{g_{k}} \nabla H_{k}\right|_{g_{k}} \\
& \leq C C_{1}^{\prime}+C \widetilde{C}_{1,0} \widetilde{C}_{1,0}:=\widetilde{C}_{2,0} .
\end{aligned}
$$

Moreover,

$$
\begin{aligned}
\frac{\partial}{\partial t}{ }^{g} \nabla H_{k} & ={ }^{g} \nabla\left(\Delta_{g_{k}} H_{k}+\operatorname{Rm}\left(g_{k}\right) * H_{k}\right) \\
& =\left({ }^{g} \nabla-{ }^{g} \nabla\right) \Delta_{g_{k}} H_{k}+{ }^{g}{ }^{g} \nabla \Delta_{g_{k}} H_{k}+{ }^{g} \nabla \operatorname{Rm}\left(g_{k}\right) * H_{k}+\operatorname{Rm}\left(g_{k}\right) *{ }^{g} \nabla H_{k}
\end{aligned}
$$

where $\Delta_{g_{k}}$ is the Laplace operator associated to $g_{k}$. Hence

$$
\begin{aligned}
& \left|\frac{\partial}{\partial t}{ }^{g} \nabla H_{k}\right|_{g} \\
& \quad \leq\left.\left. C\right|^{g} \nabla g_{k}\right|_{g} \cdot\left|\Delta_{g_{k}} H_{k}\right|_{g_{k}}+\left.\left.C\right|^{g_{k}} \nabla \Delta_{g_{k}} H_{k}\right|_{g}+\left.\left.C\right|^{g} \nabla \operatorname{Rm}\left(g_{k}\right)\right|_{g} \cdot\left|H_{k}\right|_{g}+\left.\left.C\left|\operatorname{Rm}\left(g_{k}\right)\right|_{g} \cdot\right|^{g} \nabla H_{k}\right|_{g} \\
& \quad \leq \widetilde{C}_{2,1} .
\end{aligned}
$$


For higher derivatives we claim that

$$
\left.\left.\right|^{g} \nabla^{p} \operatorname{Ric}\left(g_{k}\right)\right|_{g} \leq\left.\left. C_{p}^{\prime \prime}\right|^{g} \nabla^{p} g_{k}\right|_{g}+C_{p}^{\prime \prime \prime},\left.\left.\quad\right|^{g} \nabla^{p} g_{k}\right|_{g}+\left.\left.\right|^{g} \nabla^{p-1} H_{k}\right|_{g} \leq \widetilde{C}_{p, 0},
$$

for all $p \geq 1$, where $C_{p}^{\prime \prime}, C_{p}^{\prime \prime \prime}$, and $\widetilde{C}_{p, 0}$ are constants independent of $k$. For $p=1$, we have proved the second inequality, so we suffice to prove the first one with $p=1$. Indeed,

$$
\begin{aligned}
\left.\left.\right|^{g} \nabla \operatorname{Ric}\left(g_{k}\right)\right|_{g} & \left.\leq C \mid{ }^{g} \nabla-{ }^{g} \nabla\right) \operatorname{Ric}\left(g_{k}\right)+\left.{ }^{g_{k}} \nabla \operatorname{Ric}\left(g_{k}\right)\right|_{g_{k}} \\
& \leq\left. C\right|^{g} \Gamma-\left.{ }^{g_{k}} \Gamma|g \cdot| \operatorname{Ric}\left(g_{k}\right)\right|_{g_{k}}+\left.\left.C\right|^{g_{k}} \nabla \operatorname{Ric}\left(g_{k}\right)\right|_{g_{k}} \\
& \leq\left.\left. C_{1}^{\prime \prime}\right|^{g} \nabla g_{k}\right|_{g}+C_{1}^{\prime \prime \prime} .
\end{aligned}
$$

Suppose the claim holds for all $p<N(N \geq 2)$, we shall show that it also holds for $p=N$. From

$$
\begin{aligned}
\left|{ }^{g} \nabla^{N} \operatorname{Ric}\left(g_{k}\right)\right|_{g} & =\left|\sum_{i=1}^{N}{ }^{g} \nabla^{N-i}\left({ }^{g} \nabla-{ }^{g} \nabla\right)^{g_{k}} \nabla^{i-1} \operatorname{Ric}\left(g_{k}\right)+{ }^{g_{k}} \nabla^{N} \operatorname{Ric}\left(g_{k}\right)\right|_{g} \\
& \leq \sum_{i=1}^{N}\left|{ }^{g} \nabla^{N-i}\left({ }^{g} \nabla-{ }^{g} \nabla\right)^{g_{k}} \nabla^{i-1} \operatorname{Ric}\left(g_{k}\right)\right|_{g}+\left.\left.\right|^{g_{k}} \nabla^{N} \operatorname{Ric}\left(g_{k}\right)\right|_{g}
\end{aligned}
$$

we estimate each term. For $i=1$, by induction and the assumptions we have

$$
\begin{aligned}
\left.\right|^{g} \nabla^{N-1}\left({ }^{g} \nabla-{ }^{g} \nabla\right) & \left.\operatorname{Ric}\left(g_{k}\right)\right|_{g} \\
& \leq\left.\left. C\right|^{g} \nabla^{N-1}\left({ }^{g} \nabla g_{k} \cdot \operatorname{Ric}\left(g_{k}\right)\right)\right|_{g} \\
& \leq C\left|\sum_{j=0}^{N-1}\left(\begin{array}{c}
N-1 \\
j
\end{array}\right){ }^{g} \nabla^{N-1-j}\left(g^{g} \nabla g_{k}\right) \cdot{ }^{g} \nabla^{j}\left(\operatorname{Ric}\left(g_{k}\right)\right)\right|_{g} \\
& \leq\left.\left.\left.\left. C \sum_{j=0}^{N-1}\left(\begin{array}{c}
N-1 \\
j
\end{array}\right)\right|^{g} \nabla^{N-j} g_{k}\right|_{g} \cdot\right|^{g} \nabla^{j} \operatorname{Ric}\left(g_{k}\right)\right|_{g} \\
& \leq\left.\left. C \sum_{i=0}^{N-1}\left(\begin{array}{c}
N-1 \\
j
\end{array}\right)\left(\left.\left.C_{j}^{\prime \prime}\right|^{g} \nabla^{j} g_{k}\right|_{g}+C_{j}^{\prime \prime \prime}\right)\right|^{g} \nabla^{N-j} g_{k}\right|_{g} \\
& \leq\left.\left. C \sum_{j=0}^{N-1}\left(\begin{array}{c}
N-1 \\
j
\end{array}\right)\left(C_{j}^{\prime \prime} \widetilde{C}_{j, 0}+C_{j}^{\prime \prime \prime}\right)\right|^{g} \nabla^{N-j} g_{k}\right|_{g} \\
& =\left.\left.C(N-1)\left(C_{0}^{\prime \prime} \widetilde{C}_{j, 0}+C_{0}^{\prime \prime \prime}\right)\right|^{g} \nabla^{N} g_{k}\right|_{g}+C \sum_{j=1}^{N-1}\left(\begin{array}{c}
N-1 \\
j
\end{array}\right)\left(C_{j}^{\prime \prime} \widetilde{C}_{j, 0}+C_{j}^{\prime \prime \prime}\right) \widetilde{C}_{N-j, 0} \\
& \leq\left. C_{N}^{\prime \prime}\right|^{g} \nabla^{N} g_{k} \mid g+C_{N}^{\prime \prime \prime} \cdot
\end{aligned}
$$


For $i \geq 2$, we have

$$
\begin{aligned}
\left|{ }^{g} \nabla^{N-i}\left({ }^{g} \nabla-{ }^{g_{k}} \nabla\right)^{g_{k}} \nabla^{i-1} \operatorname{Ric}\left(g_{k}\right)\right|_{g} & \leq C\left|{ }^{g} \nabla^{N-i}\left({ }^{g} \nabla g_{k} \cdot{ }^{g_{k}} \nabla^{i-1} \operatorname{Ric}\left(g_{k}\right)\right)\right|_{g} \\
& \leq\left.\left.\left. C \sum_{j=0}^{N-i}\left(\begin{array}{c}
N-i \\
j
\end{array}\right)\right|^{g} \nabla^{N-i-j+1} g_{k}\right|_{g} \cdot\right|^{g} \nabla^{j} .\left.g_{k} \nabla^{i-1} \operatorname{Ric}\left(g_{k}\right)\right|_{g}
\end{aligned}
$$

If $j=0$, then

$$
\left.\left.\right|^{g_{k}} \nabla^{i-1} \operatorname{Ric}\left(g_{k}\right)\right|_{g} \leq\left.\left. C_{i-1}^{\prime \prime}\right|^{g} \nabla^{i-1} g_{k}\right|_{g}+C_{i-1}^{\prime \prime \prime} \leq C_{i-1}^{\prime \prime} \widetilde{C}_{i-1,0}+C_{i-1}^{\prime \prime \prime} .
$$

Suppose in the following that $j \geq 1$. Hence

$$
\begin{aligned}
\left|{ }^{g} \nabla^{j} . g_{k} \nabla^{i-1} \operatorname{Ric}\left(g_{k}\right)\right|_{g} & =\left|\left(\left({ }^{g} \nabla-{ }^{g_{k}} \nabla\right)+{ }^{g_{k}} \nabla\right)^{j} \cdot g_{k} \nabla^{i-1} \operatorname{Ric}\left(g_{k}\right)\right|_{g} \\
& \leq\left.\left.\left.\left. C \sum_{l=0}^{j}\left(\begin{array}{l}
j \\
l
\end{array}\right)\right|^{g} \nabla^{l} g_{k}\right|_{g} \cdot\right|^{g_{k}} \nabla^{j-l+i-1} \operatorname{Ric}\left(g_{k}\right)\right|_{g} \\
& \leq C \sum_{l=0}^{j}\left(\begin{array}{l}
j \\
l
\end{array}\right) \widetilde{C}_{l, 0}\left(C_{j-l+i-1}^{\prime \prime} \widetilde{C}_{j-l+i-1,0}+C_{j-l+i-1}^{\prime \prime \prime}\right),
\end{aligned}
$$

where we make use of (5-2) from first line to second line. Combining these inequalities, we get

$$
\left.\left.\right|^{g} \nabla^{N} \operatorname{Ric}\left(g_{k}\right)\right|_{g} \leq\left.\left. C_{N}^{\prime \prime}\right|^{g} \nabla^{N} g_{k}\right|_{g}+C_{N}^{\prime \prime \prime}
$$

Similarly, we have

$$
\left.\left.\right|^{g} \nabla^{N} h_{k}\right|_{g} \leq\left.\left. C_{N}^{\prime \prime}\right|^{g} \nabla^{N} g_{k}\right|_{g}+C_{N}^{\prime \prime \prime}
$$

Since $\frac{\partial}{\partial t} g_{k}=-2 \operatorname{Ric}\left(g_{k}\right)+\frac{1}{2} h_{k}$, it follows that

$$
\begin{aligned}
\frac{\partial}{\partial t}{ }^{g} \nabla^{N} g_{k} & ={ }^{g} \nabla^{N}\left(-2 \operatorname{Ric}\left(g_{k}\right)+\frac{1}{2} h_{k}\right), \\
\left.\left.\frac{\partial}{\partial t}\right|^{g} \nabla^{N} g_{k}\right|_{g} ^{2} & \leq\left|\frac{\partial}{\partial t}{ }^{g} \nabla^{N} g_{k}\right|_{g}^{2}+\left.\left.\right|^{g} \nabla^{N} g_{k}\right|_{g} ^{2} \\
& \leq\left.\left. 8\right|^{g} \nabla^{N} \operatorname{Ric}\left(g_{k}\right)\right|_{g} ^{2}+\left.\left.\frac{1}{2}\right|^{g} \nabla^{N} h_{k}\right|_{g} ^{2}+\left.\left.\right|^{g} \nabla^{N} g_{k}\right|_{g} ^{2} \\
& \leq\left.\left.\left(1+18\left(C_{N}^{\prime \prime}\right)^{2}\right)\right|^{g} \nabla^{N} g_{k}\right|_{g} ^{2}+18\left(C_{N}^{\prime \prime}\right)^{2} .
\end{aligned}
$$

Integrating the above inequality, we get $\left.\left.\right|^{g} \nabla g_{k}\right|_{g} \leq \widetilde{C}_{N, 0}$ and therefore $\left.\left.\right|^{g} \nabla^{N} h_{k}\right|_{g} \leq \widetilde{C}_{N+1,0}$. We have proved lemma for $q=0$. When $g \geq 1$, then

$$
\frac{\partial^{q}}{\partial t^{q}}{ }^{g} \nabla^{p} g_{k}(t)=g \nabla^{p} \frac{\partial^{q-1}}{\partial t^{q-1}}\left(-2 \operatorname{Ric}\left(g_{k}(t)\right)+\frac{1}{2} h_{k}(t)\right) .
$$

Using the evolution equations for $\operatorname{Rm}\left(g_{k}(t)\right)$ and $h_{k}(t)$, combining the induction to $q$ and using the above method, we have

$$
\left|\frac{\partial^{q}}{\partial t^{q}}{ }^{g} \nabla^{p} g_{k}(t)\right|_{g}+\left|\frac{\partial^{q}}{\partial t^{q}} g^{g} \nabla^{p-1} h_{k}(t)\right|_{g} \leq \widetilde{C}_{p, q}
$$




\section{Generalization}

In this section, we generalize the main results in Sections 4 and 5 to a kind of generalized Ricci flow for which local existence has been established [He et al. 2008].

Let $\left(M, g_{i j}(x)\right)$ be an $n$-dimensional closed Riemannian manifold and let $A=\left\{A_{i}\right\}$ and $B=\left\{B_{i j}\right\}$ denote a one-form and a two-form respectively. Set $F=d A$ and $H=d B$. The authors in [He et al. 2008] proved that there exists a constant $T>0$ such that the evolution equations

$$
\begin{aligned}
\frac{\partial}{\partial t} g_{i j}(x, t) & =-2 R_{i j}(x, t)+\frac{1}{2} h_{i j}(x, t)+2 f_{j k}(x, t), \quad g_{i j}(x, 0)=g_{i j}(x), \\
\frac{\partial}{\partial t} A_{i}(x, t) & =-2 \nabla_{k} F_{i}{ }^{k}(x, t), \quad A_{i}(x, 0)=A_{i}(x), \\
\frac{\partial}{\partial t} B_{i j}(x, t) & =3 \nabla_{k} H^{k}{ }_{i j}(x, t), \quad B_{i j}(x, 0)=B_{i j}(x)
\end{aligned}
$$

has a unique smooth solution on $m \times[0, T)$, where $h_{i j}=H_{i k l} H_{j}{ }^{k l}$ and $f_{i j}=F_{i}{ }^{k} F_{j k}$. We call it $\mathrm{RF}(A, B)$. According to the definition of the adjoint operator $d^{*}$, we have

$$
\left(d^{*} F\right)_{i}=2 \nabla_{k} F_{i}{ }^{k}, \quad\left(d^{*} H\right)_{i j}=-3 \nabla_{k} H^{k}{ }_{i j},
$$

and hence

$$
\begin{aligned}
& \frac{\partial}{\partial t} F(x, t)=-d d_{g(x, t)}^{*} F=\Delta_{\mathrm{HL}, g(x, t)} F=\Delta F+\mathrm{Rm} * F, \\
& \frac{\partial}{\partial t} H(x, t)=-d d_{g(x, t)}^{*} H=\Delta_{\mathrm{HL}, g(x, t)} H=\Delta H+\mathrm{Rm} * H .
\end{aligned}
$$

They also derived the evolution equations of curvatures:

$$
\begin{aligned}
\frac{\partial}{\partial t} R_{i j k \ell}= & \Delta R_{i j k \ell}+2\left(B_{i j k \ell}-B_{i j \ell k}-B_{i \ell j k}+B_{i k j \ell}\right) \\
& -g^{p q}\left(R_{p j k \ell} R_{q i}+R_{i p k \ell} R_{q j}+R_{i j p \ell} R_{q k}+R_{i j k p} R_{q \ell}\right) \\
& +\frac{1}{4}\left[\nabla_{i} \nabla_{\ell}\left(H_{k p q} H_{j}{ }^{p q}\right)-\nabla_{i} \nabla_{k}\left(H_{j p q} H_{\ell}{ }^{p q}\right)-\nabla_{j} \nabla_{\ell}\left(H_{k p q} H_{i}^{p q}\right)+\nabla_{j} \nabla_{k}\left(H_{i p q} H_{\ell}^{p q}\right)\right] \\
& +\frac{1}{4} g^{r s}\left(H_{k p q} H_{r}^{p q} R_{i j s \ell}+H_{r p q} H_{\ell}^{p q} R_{i j k s}\right) \\
& +\nabla_{i} \nabla_{\ell}\left(F_{k}{ }^{p} F_{j p}\right)-\nabla_{i} \nabla_{k}\left(F_{j}^{p} F_{\ell p}\right)-\nabla_{j} \nabla_{\ell}\left(F_{k}^{p} F_{i p}\right)+\nabla_{j} \nabla_{k}\left(F_{i}^{p} F_{\ell p}\right) \\
& +g^{r s}\left(F_{k}{ }^{p} F_{r p} R_{i j s \ell}+F_{r}^{p} F_{\ell p} R_{i j k s}\right) .
\end{aligned}
$$

Under our notation, it can be rewritten as

$$
\begin{array}{rl}
\frac{\partial}{\partial t} \mathrm{Rm}=\Delta \mathrm{Rm}+\sum_{i+j=0} \nabla^{i} \mathrm{Rm} & * \nabla^{j} \mathrm{Rm}+\sum_{i+j=0+2} \nabla^{i} H * \nabla^{j} H+\sum_{i+j=0+2} \nabla^{i} F * \nabla^{j} F \\
& +\sum_{i+j+k=0} \nabla^{i} H * \nabla^{j} H * \nabla^{k} \mathrm{Rm}+\sum_{i+j+k=0} ! ! \nabla^{i} F * \nabla^{j} F * \nabla^{k} \mathrm{Rm} .
\end{array}
$$

As before, we have: 
Proposition 6.1. For $\operatorname{RF}(A, B)$ and any nonnegative integer $\ell$ we have

$$
\begin{array}{r}
\frac{\partial}{\partial t} \nabla^{\ell} \mathrm{Rm}=\Delta\left(\nabla^{l} \mathrm{Rm}\right)+\sum_{i+j=\ell} \nabla^{i} \mathrm{Rm} * \nabla^{j} \mathrm{Rm}+\sum_{i+j=\ell+2} \nabla^{i} H * \nabla^{j} H+\sum_{i+j=\ell+2} \nabla^{i} F * \nabla^{j} F \\
+\sum_{i+j+k=\ell} \nabla^{i} H * \nabla^{j} H * \nabla^{k} \mathrm{Rm}+\sum_{i+j+k=\ell} \nabla^{i} F * \nabla^{j} F * \nabla^{k} \mathrm{Rm} .
\end{array}
$$

In particular,

$$
\begin{aligned}
& \frac{\partial}{\partial t}\left|\nabla^{l} \mathrm{Rm}\right|^{2} \leq \Delta\left|\nabla^{l} \mathrm{Rm}\right|^{2}-2\left|\nabla^{\ell+1} \mathrm{Rm}\right|^{2}+C \sum_{i+j=\ell}\left|\nabla^{i} \mathrm{Rm}\right| \cdot\left|\nabla^{j} \mathrm{Rm}\right| \cdot\left|\nabla^{\ell} \mathrm{Rm}\right| \\
& +C \sum_{i+j=\ell+2}\left|\nabla^{i} H\right| \cdot\left|\nabla^{j} H\right| \cdot\left|\nabla^{\ell} \mathrm{Rm}\right|+C \sum_{i+j=\ell+2}\left|\nabla^{i} F\right| \cdot\left|\nabla^{j} F\right| \cdot\left|\nabla^{\ell} \mathrm{Rm}\right| \\
& +C \sum_{i+j+k=\ell}\left|\nabla^{i} H\right| \cdot\left|\nabla^{j} H\right| \cdot\left|\nabla^{k} \mathrm{Rm}\right| \cdot\left|\nabla^{\ell} \mathrm{Rm}\right|+C \sum_{i+j+k=\ell}\left|\nabla^{i} F\right| \cdot\left|\nabla^{j} F\right| \cdot\left|\nabla^{k} \mathrm{Rm}\right| \cdot\left|\nabla^{\ell} \mathrm{Rm}\right|
\end{aligned}
$$

Since $\frac{\partial}{\partial t} F=\Delta F+\mathrm{Rm} * F$ it follows that

$$
\begin{aligned}
\frac{\partial}{\partial t} \nabla F & =\nabla \frac{\partial}{\partial t} F+F * \nabla(\mathrm{Rm}+H * H+F * F) \\
& =\nabla(\Delta F+\mathrm{Rm} * F)+F * \nabla \mathrm{Rm}+F * H * \nabla H+F * F * \nabla F \\
& =\Delta(\nabla F)+\nabla \mathrm{Rm} * F+\mathrm{Rm} * \nabla F+F * H * \nabla H+F * F * \nabla F .
\end{aligned}
$$

It can be expressed as

$$
\begin{aligned}
\frac{\partial}{\partial t} \nabla F=\Delta(\nabla F)+ & \sum_{i+j=1} \nabla^{i} F * \nabla^{j} \mathrm{Rm} \\
& +\sum_{i+j+k=1} \nabla^{i} F * \nabla^{j} F * \nabla^{k} F+\sum_{i=0}^{1-1} \sum_{j=0}^{1-i} \nabla^{i} F * \nabla^{j} H * \nabla^{1-i-j} H .
\end{aligned}
$$

More generally, we can show:

Proposition 6.2. $\operatorname{For} \operatorname{RF}(A, B)$ and any positive integer $\ell$ we have

$$
\begin{aligned}
\frac{\partial}{\partial t} \nabla^{\ell} F=\Delta\left(\nabla^{\ell} F\right) & +\sum_{i+j=\ell} \nabla^{i} F * \nabla^{j} \mathrm{Rm} \\
& +\sum_{i+j+k=\ell} \nabla^{i} F * \nabla^{j} F * \nabla^{k} F+\sum_{i=0}^{\ell-1} \sum_{j=0}^{\ell-i} \nabla^{i} F * \nabla^{j} H * \nabla^{\ell-i-j} H .
\end{aligned}
$$

In particular,

$$
\begin{aligned}
\frac{\partial}{\partial t}\left|\nabla^{\ell} F\right|^{2} \leq & \Delta\left|\nabla^{\ell} F\right|^{2}-2\left|\nabla^{\ell+1} F\right|^{2}+C \sum_{i+j=\ell}\left|\nabla^{i} F\right| \cdot\left|\nabla^{j} \mathrm{Rm}\right| \cdot\left|\nabla^{\ell} F\right| \\
& +C \sum_{i+j+k=\ell}\left|\nabla^{i} F\right| \cdot\left|\nabla^{j} F\right| \cdot\left|\nabla^{k} F\right| \cdot\left|\nabla^{l} F\right|+C \sum_{i=0 j=0}^{\ell-1 \ell-i}\left|\nabla^{i} F\right| \cdot\left|\nabla^{j} H\right| \cdot\left|\nabla^{\ell-i-j} H\right| \cdot\left|\nabla^{\ell} F\right| .
\end{aligned}
$$


Similarly, we obtain:

Proposition 6.3. For $\operatorname{RF}(A, B)$ and any positive integer $l$ we have

$$
\begin{array}{rl}
\frac{\partial}{\partial t} \nabla^{\ell} H=\Delta\left(\nabla^{\ell} H\right)+\sum_{i+j=\ell} \nabla^{i} H & * \nabla^{j} \mathrm{Rm} \\
& +\sum_{i+j+k=\ell} \nabla^{i} H * \nabla^{j} H * \nabla^{k} H+\sum_{i=0}^{\ell-1} \sum_{j=0}^{\ell-i} \nabla^{i} H * \nabla^{j} F * \nabla^{\ell-i-j} F .
\end{array}
$$

In particular,

$$
\begin{aligned}
\frac{\partial}{\partial t}\left|\nabla^{\ell} H\right|^{2} & \leq \Delta\left|\nabla^{\ell} H\right|^{2}-2\left|\nabla^{\ell+1} H\right|^{2}+C \sum_{i+j=\ell}\left|\nabla^{i} H\right| \cdot\left|\nabla^{j} \mathrm{Rm}\right| \cdot\left|\nabla^{\ell} H\right| \\
& +C \sum_{i+j+k=\ell}\left|\nabla^{i} H\right| \cdot\left|\nabla^{j} H\right| \cdot\left|\nabla^{k} H\right| \cdot\left|\nabla^{\ell} H\right|+C \sum_{i=0}^{\ell-1} \sum_{j=0}^{\ell-i}\left|\nabla^{i} H\right| \cdot\left|\nabla^{j} F\right| \cdot\left|\nabla^{\ell-i-j} F\right| \cdot\left|\nabla^{\ell} H\right| .
\end{aligned}
$$

From the evolution inequalities

$$
\begin{aligned}
& \frac{\partial}{\partial t}|H|^{2} \leq \Delta|H|^{2}-2|\nabla H|^{2}+C \cdot|\mathrm{Rm}| \cdot|H|^{2}, \\
& \frac{\partial}{\partial t}|F|^{2} \leq \Delta|F|^{2}-2|\nabla F|^{2}+C \cdot|\mathrm{Rm}| \cdot|F|^{2},
\end{aligned}
$$

the following theorem is obvious.

Theorem 6.4. Suppose that $(g(x, t), H(x, t), F(x, t))$ is a solution to $\operatorname{RF}(A, B)$ on a compact manifold $M^{n}$ for a short time $0 \leq t \leq T$ and $K_{1}, K_{2}, K_{3}$ are arbitrary given nonnegative constants. Then there exists a constant $C_{n}$ depending only on $n$ such that if

$$
|\operatorname{Rm}(x, t)|_{g(x, t)} \leq K_{1}, \quad|H(x)|_{g(x)} \leq K_{2}, \quad|F(x)|_{g(x)} \leq K_{3}
$$

for all $x \in M$ and $t \in[0, T]$, then

$$
|H(x, t)|_{g(x, t)} \leq K_{2} e^{C_{n} K_{1} t}, \quad|F(x, t)|_{g(x, t)} \leq K_{3} e^{C_{n} K_{1} t},
$$

for all $x \in M$ and $t \in[0, T]$.

Parallel to Theorem 4.6, we can prove:

Theorem 6.5. Suppose that $(g(x, t), H(x, t), F(x, t))$ is a solution to $\operatorname{RF}(A, B)$ on a compact manifold $M^{n}$ and $K$ is an arbitrary given positive constant. Then for each $\alpha>0$ and each integer $m \geq 1$ there exists a constant $C_{m}$ depending on $m, n, \max \{\alpha, 1\}$, and $K$ such that if

$$
|\operatorname{Rm}(x, t)|_{g(x, t)} \leq K, \quad|H(x)|_{g(x)} \leq K, \quad|F(x)|_{g(x)} \leq K
$$

for all $x \in M$ and $t \in[0, \alpha / K]$, then

$$
\left|\nabla^{m-1} \operatorname{Rm}(x, t)\right|_{g(x, t)}+\left|\nabla^{m} H(x, t)\right|_{g(x, t)}+\left|\nabla^{m} F(x, t)\right|_{g(x, t)} \leq \frac{C_{m}}{t^{\frac{m}{2}}},
$$

for all $x \in M$ and $t \in(0, \alpha / K]$. 
We can also establish the corresponding compactness theorem for $\operatorname{RF}(A, B)$. We omit the detail since the proof is close to the proof in Section 5. In the forthcoming paper, we will consider the BBS estimates for complete noncompact Riemannian manifolds.

\section{Acknowledgment}

The author thanks his advisor, Professor Shing-Tung Yau, for helpful discussions. The author expresses his gratitude to Professor Kefeng Liu for his interest in this work and for his numerous help in mathematics. He also thanks Valentino Tosatti and Jeff Streets for several useful conversations.

\section{References}

[Bakas 2007] I. Bakas, "Renormalization group equations and geometric flows", preprint, 2007. arXiv hep-th/0702034v1

[Cao and Zhu 2006] H.-D. Cao and X.-P. Zhu, "A complete proof of the Poincaré and geometrization conjectures: application of the Hamilton-Perelman theory of the Ricci flow", Asian J. Math. 10:2 (2006), 165-492. MR 2008d:53090 Zbl 1200.53057

[Chow and Knopf 2004] B. Chow and D. Knopf, The Ricci flow: an introduction, Mathematical Surveys and Monographs 110, American Mathematical Society, Providence, RI, 2004. MR 2005e:53101 Zbl 1086.53085

[Chow et al. 2006] B. Chow, P. Lu, and L. Ni, Hamilton's Ricci flow, Graduate Studies in Mathematics 77, American Mathematical Society, Providence, RI, 2006. MR 2008a:53068 Zbl 1118.53001

[Chow et al. 2007] B. Chow, S.-C. Chu, D. Glickenstein, C. Guenther, J. Isenberg, T. Ivey, D. Knopf, P. Lu, F. Luo, and L. Ni, The Ricci flow: techniques and applications, I: Geometric aspects, Mathematical Surveys and Monographs 135, American Mathematical Society, Providence, RI, 2007. MR 2008f:53088 Zbl 1157.53034

[Chow et al. 2008] B. Chow, S.-C. Chu, D. Glickenstein, C. Guenther, J. Isenberg, T. Ivey, D. Knopf, P. Lu, F. Luo, and L. $\mathrm{Ni}$, The Ricci flow: techniques and applications, II: Analytic aspects, Mathematical Surveys and Monographs 144, American Mathematical Society, Providence, RI, 2008. MR 2008j:53114 Zbl 1157.53035

[Chow et al. 2010] B. Chow, S.-C. Chu, D. Glickenstein, C. Guenther, J. Isenberg, T. Ivey, D. Knopf, P. Lu, F. Luo, and L. Ni, The Ricci flow: techniques and applications, III: Geometric-analytic aspects, Mathematical Surveys and Monographs 163, American Mathematical Society, Providence, RI, 2010. MR 2011g:53142 Zbl 1216.53057

[Gegenberg and Kunstatter 2004] J. Gegenberg and G. Kunstatter, "Using 3D string-inspired gravity to understand the Thurston conjecture", Classical Quantum Gravity 21:4 (2004), 1197-1207. MR 2004j:53083 Zbl 1046.83024

[Hamilton 1982] R. S. Hamilton, "Three-manifolds with positive Ricci curvature”, J. Differential Geom. 17:2 (1982), 255-306. MR 84a:53050 Zbl 0504.53034

[Hamilton 1995] R. S. Hamilton, "A compactness property for solutions of the Ricci flow", Amer. J. Math. 117:3 (1995), 545-572. MR 96c:53056 Zbl 0840.53029

[He et al. 2008] C.-L. He, S. Hu, D.-X. Kong, and K. Liu, "Generalized Ricci flow, I: Local existence and uniqueness", pp. 151-171 in Topology and physics, edited by K. Lin et al., Nankai Tracts Math. 12, World Scientific, Hackensack, NJ, 2008. MR 2010k:53098 Zbl 1182.35145

[Kleiner and Lott 2008] B. Kleiner and J. Lott, "Notes on Perelman's papers", Geom. Topol. 12:5 (2008), 2587-2855. MR 2010h:53098 Zbl 1204.53033

[Morgan and Tian 2007] J. Morgan and G. Tian, Ricci flow and the Poincaré conjecture, Clay Mathematics Monographs 3, American Mathematical Society, Providence, RI, 2007. MR 2008d:57020 Zbl 1179.57045

[Oliynyk et al. 2006] T. Oliynyk, V. Suneeta, and E. Woolgar, "A gradient flow for worldsheet nonlinear sigma models", Nuclear Phys. B 739:3 (2006), 441-458. MR 2006m:81185 Zbl 1109.81058

[Perelman 2002] G. Perelman, "The entropy formula for the Ricci flow and its geometric applications", preprint, 2002. Zbl 1130.53001 arXiv math.DG/0211159

[Shi 1989] W.-X. Shi, "Deforming the metric on complete Riemannian manifolds", J. Differential Geom. 30:1 (1989), $223-301$. MR 90i:58202 Zbl 0676.53044 
[Streets 2007] J. D. Streets, Ricci Yang-Mills flow, thesis, Duke University, Durham, NC, 2007, Available at http://tinyurl.com/ Streets-JD-2007-thesis. MR 2709943

[Streets 2008] J. D. Streets, "Regularity and expanding entropy for connection Ricci flow", J. Geom. Phys. 58:7 (2008), 900-912. MR 2009f:53105 Zbl 1144.53326

[Young 2008] A. N. Young, Modified Ricci flow on a principal bundle, thesis, University of Texas, Austin, TX, 2008, Available at http://tinyurl.com/Young-AN-2008-thesis. MR 2712036

Received 22 Sep 2010. Revised 4 Aug 2011. Accepted 27 Sep 2011.

YI LI: yli@math.jhu.edu

Department of Mathematics, Johns Hopkins University, 3400 N. Charles Street, Baltimore, MD 21218, United States 


\title{
Analysis \& PDE
}

\author{
msp.berkeley.edu/apde
}

EDITORS

EDITOR-IN-CHIEF

Maciej Zworski

University of California

Berkeley, USA

BOARD OF EDITORS

\begin{tabular}{|c|c|c|c|}
\hline Michael Aizenman & $\begin{array}{l}\text { Princeton University, USA } \\
\text { aizenman@math.princeton.edu }\end{array}$ & Nicolas Burq & $\begin{array}{l}\text { Université Paris-Sud 11, France } \\
\text { nicolas.burq@math.u-psud.fr }\end{array}$ \\
\hline Luis A. Caffarelli & $\begin{array}{l}\text { University of Texas, USA } \\
\text { caffarel@ math.utexas.edu }\end{array}$ & un-Yung Alice Chang & $\begin{array}{l}\text { Princeton University, USA } \\
\text { chang@ math.princeton.edu }\end{array}$ \\
\hline Michael Christ & $\begin{array}{l}\text { University of California, Berkeley, USA } \\
\text { mchrist@math.berkeley.edu }\end{array}$ & Charles Fefferman & $\begin{array}{l}\text { Princeton University, USA } \\
\text { cf@math.princeton.edu }\end{array}$ \\
\hline Ursula Hamenstaedt & $\begin{array}{l}\text { Universität Bonn, Germany } \\
\text { ursula@math.uni-bonn.de }\end{array}$ & Nigel Higson & $\begin{array}{l}\text { Pennsylvania State Univesity, USA } \\
\text { higson@math.psu.edu }\end{array}$ \\
\hline Vaughan Jones & $\begin{array}{l}\text { University of California, Berkeley, USA } \\
\text { vfr@math.berkeley.edu }\end{array}$ & Herbert Koch & $\begin{array}{l}\text { Universität Bonn, Germany } \\
\text { koch@math.uni-bonn.de }\end{array}$ \\
\hline Izabella Laba & $\begin{array}{l}\text { University of British Columbia, Canada } \\
\text { ilaba@math.ubc.ca }\end{array}$ & Gilles Lebeau & $\begin{array}{l}\text { Université de Nice Sophia Antipolis, France } \\
\text { lebeau@unice.fr }\end{array}$ \\
\hline László Lempert & $\begin{array}{l}\text { Purdue University, USA } \\
\text { lempert@math.purdue.edu }\end{array}$ & Richard B. Melrose & $\begin{array}{l}\text { Massachussets Institute of Technology, USA } \\
\text { rbm@math.mit.edu }\end{array}$ \\
\hline Frank Merle & $\begin{array}{l}\text { Université de Cergy-Pontoise, France } \\
\text { Frank.Merle@u-cergy.fr }\end{array}$ & William Minicozzi II & $\begin{array}{l}\text { Johns Hopkins University, USA } \\
\text { minicozz@ math.jhu.edu }\end{array}$ \\
\hline Werner Müller & $\begin{array}{l}\text { Universität Bonn, Germany } \\
\text { mueller@math.uni-bonn.de }\end{array}$ & Yuval Peres & $\begin{array}{l}\text { University of California, Berkeley, USA } \\
\text { peres@stat.berkeley.edu }\end{array}$ \\
\hline Gilles Pisier & $\begin{array}{l}\text { Texas A\&M University, and Paris } 6 \\
\text { pisier@math.tamu.edu }\end{array}$ & Tristan Rivière & $\begin{array}{l}\text { ETH, Switzerland } \\
\text { riviere@math.ethz.ch }\end{array}$ \\
\hline Igor Rodnianski & $\begin{array}{l}\text { Princeton University, USA } \\
\text { irod@math.princeton.edu }\end{array}$ & Wilhelm Schlag & $\begin{array}{l}\text { University of Chicago, USA } \\
\text { schlag@math.uchicago.edu }\end{array}$ \\
\hline Sylvia Serfaty & $\begin{array}{l}\text { New York University, USA } \\
\text { serfaty@ cims.nyu.edu }\end{array}$ & Yum-Tong Siu & $\begin{array}{l}\text { Harvard University, USA } \\
\text { siu@math.harvard.edu }\end{array}$ \\
\hline Terence Tao & $\begin{array}{l}\text { University of California, Los Angeles, USA } \\
\text { tao@math.ucla.edu }\end{array}$ & Michael E. Taylor & $\begin{array}{l}\text { Univ. of North Carolina, Chapel Hill, USA } \\
\text { met@math.unc.edu }\end{array}$ \\
\hline Gunther Uhlmann & $\begin{array}{l}\text { University of Washington, USA } \\
\text { gunther@math.washington.edu }\end{array}$ & András Vasy & $\begin{array}{l}\text { Stanford University, USA } \\
\text { andras@math.stanford.edu }\end{array}$ \\
\hline an Virgil Voiculescu & $\begin{array}{l}\text { University of California, Berkeley, USA } \\
\text { dvv@ math.berkeley.edu }\end{array}$ & Steven Zelditch & $\begin{array}{l}\text { Northwestern University, USA } \\
\text { zelditch@math.northwestern.edu }\end{array}$ \\
\hline
\end{tabular}

\section{PRODUCTION}

contact@msp.org

Silvio Levy, Scientific Editor

Sheila Newbery, Senior Production Editor

See inside back cover or msp.berkeley.edu/apde for submission instructions.

The subscription price for 2012 is US \$140/year for the electronic version, and \$240/year for print and electronic. Subscriptions, requests for back issues from the last three years and changes of subscribers address should be sent to Mathematical Sciences Publishers, Department of Mathematics, University of California, Berkeley, CA 94720-3840, USA.

Analysis \& PDE, at Mathematical Sciences Publishers, Department of Mathematics, University of California, Berkeley, CA 94720-3840 is published continuously online. Periodical rate postage paid at Berkeley, CA 94704, and additional mailing offices.

APDE peer review and production are managed by EditFLOW ${ }^{\mathrm{TM}}$ from Mathematical Sciences Publishers.

PUBLISHED BY

mathematical sciences publishers

http://msp.org/

A NON-PROFIT CORPORATION

Typeset in IATEX

Copyright $(2012$ by Mathematical Sciences Publishers 


\section{ANALYSIS \& PDE}

\section{Volume $5 \quad$ No. $4 \quad 2012$}

On the global well-posedness of energy-critical Schrödinger equations in curved spaces

Alexandru D. IONESCU, BenOIT PAusader and Gigliola StafFilani

Generalized Ricci flow, I: Higher-derivative estimates for compact manifolds

YI LI

Smooth type II blow-up solutions to the four-dimensional energy-critical wave equation

Matthieu Hillairet and PierRe RaphaËL

Nonconcentration in partially rectangular billiards

LUC Hillairet and JEREMY L. MARZUOLA

Global well-posedness and scattering for the defocusing quintic NLS in three dimensions ROWAN KILLIP and MONICA VIŞAN 